\title{
Uncovering the fatigue damage initiation and progression in uni-directional non-crimp fabric reinforced polyester composite
}

\author{
Jespersen, Kristine Munk; Glud, Jens A.; Zangenberg, Jens; Hosoi, Atsushi; Kawada, Hiroyuki; \\ Mikkelsen, Lars Pilgaard
}

\author{
Published in: \\ Composites - Part A: Applied Science and Manufacturing
}

Link to article, DOI:

10.1016/j.compositesa.2018.03.002

Publication date:

2018

Document Version

Peer reviewed version

Link back to DTU Orbit

Citation (APA):

Jespersen, K. M., Glud, J. A., Zangenberg, J., Hosoi, A., Kawada, H., \& Mikkelsen, L. P. (2018). Uncovering the fatigue damage initiation and progression in uni-directional non-crimp fabric reinforced polyester composite. Composites - Part A: Applied Science and Manufacturing, 109, 481-497.

https://doi.org/10.1016/j.compositesa.2018.03.002

\section{General rights}

Copyright and moral rights for the publications made accessible in the public portal are retained by the authors and/or other copyright owners and it is a condition of accessing publications that users recognise and abide by the legal requirements associated with these rights.

- Users may download and print one copy of any publication from the public portal for the purpose of private study or research.

- You may not further distribute the material or use it for any profit-making activity or commercial gain

- You may freely distribute the URL identifying the publication in the public portal 


\title{
Uncovering the fatigue damage initiation and progression in uni-directional non-crimp fabric reinforced polyester composite
}

\author{
Kristine M. Jespersen ${ }^{\mathrm{a}, *}$, Jens A. Glud ${ }^{\mathrm{b}}$, Jens Zangenberg ${ }^{\mathrm{c}}$, Atsushi Hosoi ${ }^{\mathrm{d}}$, Hiroyuki \\ Kawada $^{\mathrm{d}}$, Lars P. Mikkelsen ${ }^{\mathrm{a}}$ \\ ${ }^{a}$ Department of Wind Energy, Section of Composites and Materials Mechanics, Technical University \\ of Denmark, Fredriksborgvej 399, 4000 Roskilde, Denmark \\ ${ }^{b}$ Department of Mechanical and Manufacturing Engineering, Aalborg University, Fibigerstraede 16, \\ 9220 Aalborg Oest, Denmark \\ ${ }^{c}$ LM Wind Power Blades, Composite Mechanics, Jupitervej 6, 6000 Kolding, Denmark \\ ${ }^{d}$ Department of Applied Mechanics and Aerospace Engineering, Waseda University, 3-4-1, Okubo, \\ Shinjuku-ku, Tokyo 169-8555, Japan
}

\begin{abstract}
The current work studies the fatigue damage initiation and progression in a quasiunidirectional non-crimp fabric based fibre composite used for wind turbine blades. This is done by combining in-situ transilluminated white light imagining (TWLI) with exsitu X-ray computed tomography $(\mathrm{CT})$ experiments along with tension clamp X-ray CT experiments. TWLI is used to monitor the off-axis cracks in the thin supporting backing fibre bundles present in quasi-UD composites, and a crack counting algorithm is applied to automatically count the cracks in images obtained in-situ during fatigue testing. It is found that off-axis cracks not only initiate at the specimen edges but also at isolated locations inside the specimen, which could be related to the microstructural features. In addition, a clear effect of strain level on the measured off-axis crack density is observed. From the X-ray CT experiments, it is found that the UD fibre fractures initiate and progress from regions where the off-axis backing fibre bundles are 'in contact' with a UD fibre bundle. Damage is seen to first initiate at a cross-over region of the backing fibre bundles, and later at a region with only one backing fibre bundle. In addition, applying tension to the specimen during X-ray CT scanning is found to reveal additional UD
\end{abstract}

\footnotetext{
* Corresponding author.

E-mail address: kmun@dtu.dk (K.M. Jespersen).
} 
fibre fractures that are not visible in scans performed the unloaded state. With load applied, a significant number of UD fibre fractures were observed earlier in the fatigue life than expected. Based on the observations of the study a damage progression scheme is presented for quasi-UD fibre composites.

Keywords: A. Polymer Matrix Composites (PMCs), A. Glass fibres, B. Fracture, D. Non-destructive testing

\section{Introduction}

Due to their high specific stiffness, strength, and fatigue resistance, fibre composites are increasingly used for structural purposes in structures like airplanes, cars and wind turbine blades. Especially for wind turbine blades materials, fatigue is a great concern due to the high number of load cycles caused by the variation in the wind and the oscillating gravitational loads coming from the blade rotation. More specifically, a wind turbine blade is usually designed to last 20-30 years, which corresponds to more than $10^{8}$ load cycles [1, 2]. Composite and sandwich materials are used to obtain as light and long blades as possible and the cross-section of a blade is carefully designed in a way that fits the loads present. As a result, the spars that carry the main fatigue loads almost only experience axial loads are made from fibre composites where most of the fibres are oriented in the direction of the load. For this purpose, uni-directional (UD) non-crimp fabric (NCF) based composites are commonly used [1, 3, 4]. For these UD NCFs, the UD fibres (aligned in the axial blade direction) are arranged in fibre bundles that are stitched to a thin layer of supporting backing fibre bundles oriented in an off-axis direction. Due to the presence of a small fraction of off-axis fibres, this type of composite have also been referred to as quasi-UD NCF composites and will be named so in the rest of this paper.

\subsection{Designing against fatigue}

The currently used methods to design against fatigue, such as constant life diagrams based on coupon testing, have been adopted from steels although the damage 
mechanisms are fundamentally different [1-3]. Due to the lack of understanding of the fatigue damage mechanisms of composites, a lot of uncertainties are present in this design method [2] resulting in numerous safety factors in the design. For example, fatigue tests are usually accelerated to save time by testing at a higher strain level and frequency than what is present in the actual structure. However, studies have shown an influence of the strain level during fatigue on the saturation crack density [5]7] and even on the damage mechanisms such as delamination behaviour [7]. Uncertainties in the test methods is also related to it being difficult to test the quasi-UD composites used for wind turbine blades since they are much stronger in the axial direction than in shear. As a consequence, they tend to fail in the tab rather than in the gauge section even for the butterfly shaped test geometry optimised for testing quasi-UD composites [8]. Hence, the obtained fatigue life from coupon testing most likely give conservative values. To be able to establish new design methods that are better suited for fibre composites, it is necessary to first understand the underlying fatigue damage mechanisms of the materials.

\subsection{Fatigue damage mechanisms of fibre composites}

The initiation and progression of damage during fatigue loading of fibre composites is a complex matter. During fatigue loading matrix cracking, fibre/matrix debonding, and fibre fractures occur and interact with one another gradually causing the stiffness and strength of the material to degrade. A schematic of a typical stiffness degradation curve for a fibre composite subjected to fatigue loading is illustrated by the black curve in Fig. 1. Reifsnider and Jamison [9, 10] suggested to split the stiffness degradation into three main stages: the initial rapid stiffness degradation (stage I), the stable stiffness degradation (stage II), and the final stage where localisation and final failure occurs (stage III), as also marked in Fig. 1. The mechanisms that occur during these stages depend highly on the composite lay-up [9, 10]. Several studies [5-7, 9]11] have considered the fatigue damage mechanisms of prepreg based laminated composites with a somewhat homogeneous distribution of fibres within each layer, which are commonly 
used in the aerospace industry. In contrast to one layer of these prepreg based composites, the quasi-UD NCF composites used for wind turbine blades have a fibre bundle structure, a small portion $(\sim 10 \%)$ of off-axis backing fibre bundles within each layer, and uses stitching thread to tie the bundles together. As a result, the damage mechanisms for quasi-UD composites are different from the prepreg based composites. Studies have also been carried out on damage progression in NCF composites [12 20], however the damage mechanisms are still not well understood. In the case of the considered quasi-UD NCF composites, the main damage mechanisms observed so far are off-axis cracking in the thin backing fibre bundles and fibre fractures in the load carrying UD fibre bundles [18, 19]. However, the connection between these mechanisms and the order in which they occur has not yet been sufficiently clarified, and is the focus of the current study.

Previous studies [18, 19] using X-ray CT highlighted the importance of taking the 3D aspect into account when considering the UD fibre fractures of quasi-UD NCF based composites. X-ray CT has been successfully used to monitor damage in fibre composites in several studies [18, 19, 21 34], however it has the general disadvantage that cracks with an opening smaller than the resolution will not be visible (usually the voxel size is larger than $1 \mu \mathrm{m})$. As a result, X-ray CT studies generally consider small specimens to obtain sufficient image resolution. However, downsizing a fatigue test specimen excessively has been found to influence the stiffness degradation due to the edge effect [20] and furthermore for quasi-UD composites the considered volume might not be representative due to the fibre bundle structure. Therefore, a big challenge when using X-ray CT for this purpose, is the compromise between considering a representative volume unaffected by the edge effect and obtaining sufficient image resolution.

To overcome these challenges, the current study combines X-ray CT experiments with transilluminated white light imaging (TWLI), which is well suited for observing the off-axis cracks in transparent composites during the early fatigue life (see Fig. 1). An automatic crack counting algorithm [35] is used to obtain the crack density as a function of the number of fatigue load cycles. This not only has the advantage of saving 
time, but also reduces the 'human factor' by comparing the crack densities counted using the same input parameters to the crack counting algorithm for all the tests. The crack density is defined relative to the backing fibre bundle structure, which is obtained by large field of view X-ray CT experiments. The fatigue tests are performed at initial strain levels between $0.25 \%$ and $0.8 \%$ strain. In addition, ex-situ X-ray CT is used to monitor progression of the UD fibre fractures and a tension clamp solution used during X-ray CT scanning to enhance the crack visibility at two points during the fatigue life as also illustrated in Fig. 1.

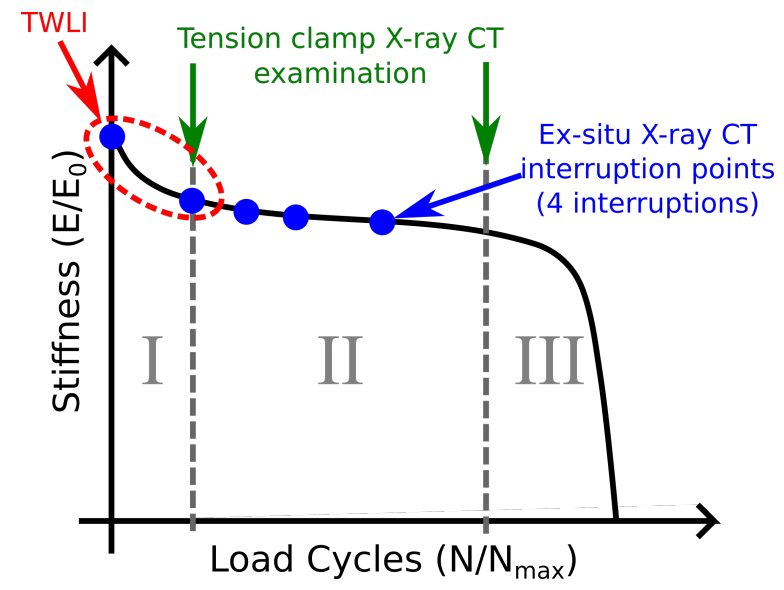

Figure 1: Schematic of a typical stiffness degradation curve for a fibre composite subjected to fatigue loading including explanation of experiments conducted in the current study.

\section{Experimental methods}

\subsection{Composite material and specimen geometry}

The current study considers a glass fibre composite made from two layers of quasiUD non-crimp fabric (UD1322) infused with a polyester matrix by vacuum assisted resin transfer moulding. The fabric consists of parallel bundles of UD fibres stitched to a layer of thin supporting $\pm 80^{\circ}$ backing fibre bundles. Table 1 shows the specifications of the used fabric, which was also used in [17, 18]. The backing fibre bundles were removed from one of the layers in the gauge section of the composite resulting in the layup $[0 / b, 0 / b] \rightarrow[0 / b, 0]$ in the considered region. Here " 0 " indicate the UD bundle 
side and "b" the backing bundle side of the fabric. The thickness of the final composite was $2 \mathrm{~mm}$ and the average fibre volume fraction was $V_{f} \approx 0.55$.

Table 1: Fabric specification (UD1322)

\begin{tabular}{lllll}
\hline Category & Unit & Axial fibres & Backing fibres & Stitching thread \\
\hline Material & - & $\mathrm{H}+$ glass & E-glass & Polyester \\
Area density & {$\left[\mathrm{g} / \mathrm{m}^{2}\right]$} & 1322 & 60 & 15 \\
Linear density & {$[\mathrm{tex}]$} & 2400 & 68 & 7.6 \\
Avg. filament dia. & {$[\mu \mathrm{m}]$} & 17 or 24 & 9 & $\mathrm{~N} / \mathrm{A}$ \\
\hline
\end{tabular}

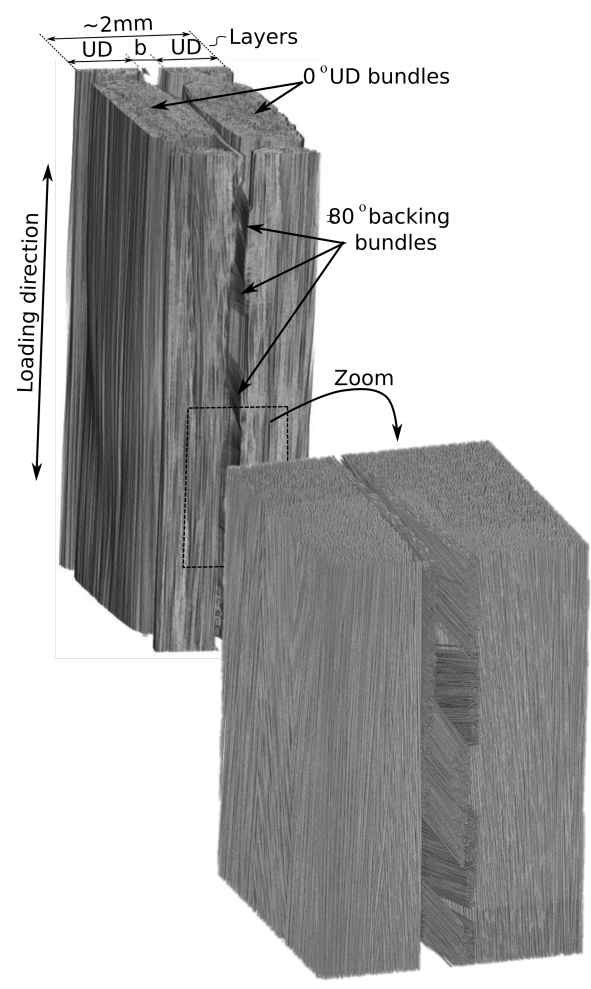

Figure 2: Example of bundle structure obtained by X-ray CT

Fig. 2 shows a $3 \mathrm{D}$ rendering of the composite obtained by X-ray CT where the matrix has been rendered invisible to illustrate the bundle architecture. It is seen that due to the fibre bundle structure there are regions of high fibre volume fraction and other regions where there are no fibres at all. Fig. 3 a shows a illustration of the layup 
and Fig. 3b shows a sketch of one quasi-UD layer with backing. It is seen that the backing bundles (marked in green) are crossing over one another at some locations.

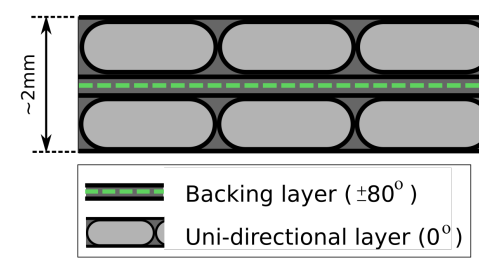

a) Composite lay-up

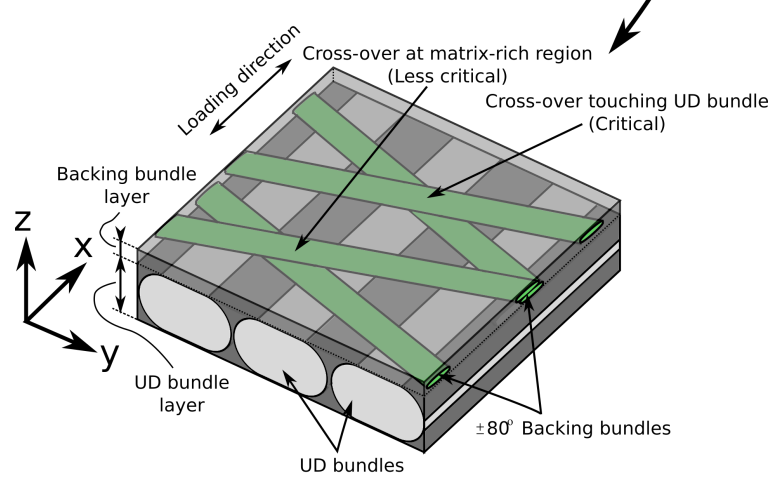

b) One UD fabric layer with backing

Figure 3: Schematic of (a) the composite layup and (b) one layer of fabric.

A plate of the quasi-UD composite was manufactured, tabs glued on, and cut into $250 \mathrm{~mm}$ long specimens with the geometry shown in Fig. 4. The test specimens were cut out so that the region where one backing layer had been removed was located in the gauge section of the test specimens. The used specimen geometry was a downscaled version of the butterfly geometry [8] commonly used to test UD composites in fatigue. This specific specimen geometry was designed with the purpose of combining the advantages of the transilluminated white light imaging (TWLI) [6, 35] and X-ray CT [18, 19] techniques to study both the fatigue damage initiation and progression throughout the fatigue life. The best resolution in X-ray CT is obtained when the cross-section of the specimen is as small as possible. However, a previous study [20] showed that the edge effect had a great influence on the results for a sample width of $5 \mathrm{~mm}$. Therefore, the specimen geometry used in this study (Fig. 4) was designed as a compromise between the full size butterfly specimen geometry [8, 18] and the small 
samples earlier considered in [20], resulting in a width of $10 \mathrm{~mm}$ in the gauge section.

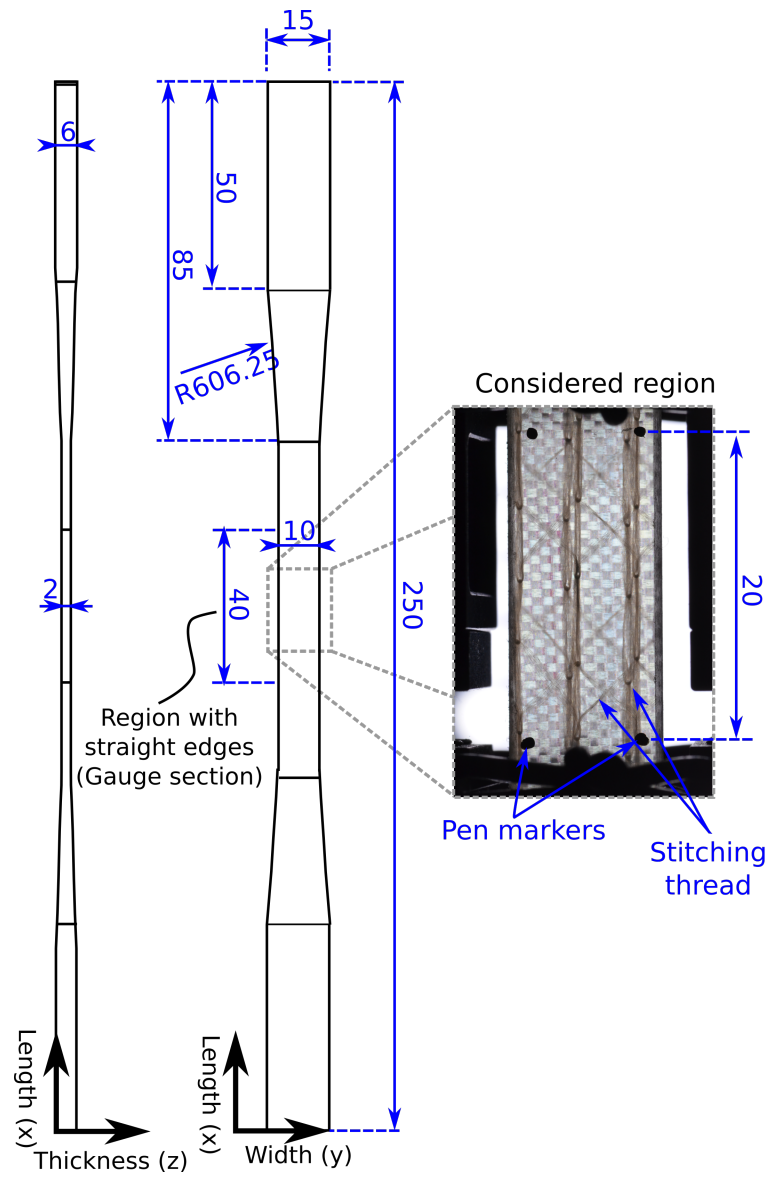

Figure 4: Specimen geometry used for fatigue test. A photo of the considered region is shown with indications of the pen markers used for the automatic crack counting algorithm.

\subsection{Fatigue testing and off-axis crack monitoring}

Tension-tension $(\mathrm{R}=0.1)$ fatigue tests were carried out in load control with a sinusodial waveform at initial strain levels in the range $\varepsilon_{\max }=0.25-0.8 \%$. Before the cyclic loading, the test specimen was loaded up to first $90 \%$ of the desired strain and subsequently up to $0.25 \%$ strain in order to firstly precondition the fatigue test setup and secondly to find the initial material stiffness in the strain range $0.05-0.25 \%$, which is in accordance with the standard ISO 527 for fibre reinforced polymers. This material stiffness determine the necessary load required to give a certain desired initial strain 
level. Thereby, the fatigue test can be performed in load control giving a specific strain level during the initial cyclic fatigue loads. Table 2 shows a summary of the 12 tests carried out. The strain was monitored using two extensometers as seen in Fig. 5. The extensometers were mounted on the edge of the specimen to be able to measure the strain in the considered region also shown in Fig. 5 and at the same time monitor the sample with a camera. To make it possible to use the automatic crack counting algorithm explained later in Section 3, pen markers were drawn around the centre of the sample with a $20 \mathrm{~mm}$ distance in between in the length direction, as also marked in the photo of the "considered region" shown in Fig. 4. The vertical and crossing lines visible in the photograph are the stitching threads and the checkerboard pattern is the peel-ply present on the back of the specimen (facing away from the camera).

Table 2: Overview of performed fatigue tests

\begin{tabular}{llll}
\hline Sample ID & Strain & $\begin{array}{c}\text { Tested } \\
\text { cycles }\end{array}$ & $\begin{array}{l}\text { Test fre- } \\
\text { quency }\end{array}$ \\
\hline EPS08-1 & $0.80 \%$ & 100,000 & $5 \mathrm{~Hz}$ \\
EPS06-1 & $0.60 \%$ & 100,0001 & $5 \mathrm{~Hz}$ \\
EPS06-2 & $0.60 \%$ & 100,000 & $5 \mathrm{~Hz}$ \\
EPS06-3 & $0.60 \%$ & 100,000 & $5 \mathrm{~Hz}$ \\
EPS06-4 & $0.60 \%$ & $1,000,000$ & $5 \mathrm{~Hz}$ \\
EPS05-1 & $0.50 \%$ & 500,000 & $5 \mathrm{~Hz}$ \\
EPS05-2 & $0.50 \%$ & 500,000 & $5 \mathrm{~Hz}$ \\
EPS05-3 & $0.50 \%$ & 500,000 & $5 \mathrm{~Hz}$ \\
EPS04-1 & $0.40 \%$ & $2,000,000$ & $5 \mathrm{~Hz}$ \\
EPS04-2 & $0.40 \%$ & $2,000,000$ & $5 \mathrm{~Hz}$ \\
EPS04-3 & $0.40 \%$ & $2,000,000$ & $5 \mathrm{~Hz}$ \\
EPS025-1 & $0.25 \%$ & $10,000,00010 \mathrm{~Hz}$ \\
\hline
\end{tabular}

During all the performed fatigue tests, TWLI was used to monitor the sample continously. The sample was lit up by an LED lamp on the back and a Nikon D7000 
camera with a Tokina Macro 100 F2.8D lens was used to capture images during the test as shown in Fig. 5. This gave a pixel size of around $5 \mu \mathrm{m}$ in the photos. The camera was connected to a trigger box programmed to take photos at the point of max load for specific cycle numbers during the fatigue test. One photo was taken before the sample had been introduced to any load and another photo was taken after the two initial static tests. During the fatigue test 100 photos were taken during each cycle decade. Hence, the trigger box was programmed to take 100 photos during the first 100 cycles, every 10th cycle up to 1000 cycles, every 100th cycle up to 10000 cycles, and so on. However, if the stiffness experienced a sudden drop (more than 1\%), the camera was automatically triggered to take photos with a higher frequency. This approach was chosen in order to capture the damage intiation and progression without getting an overload of data. The TWLI data can also be downloaded online [36].

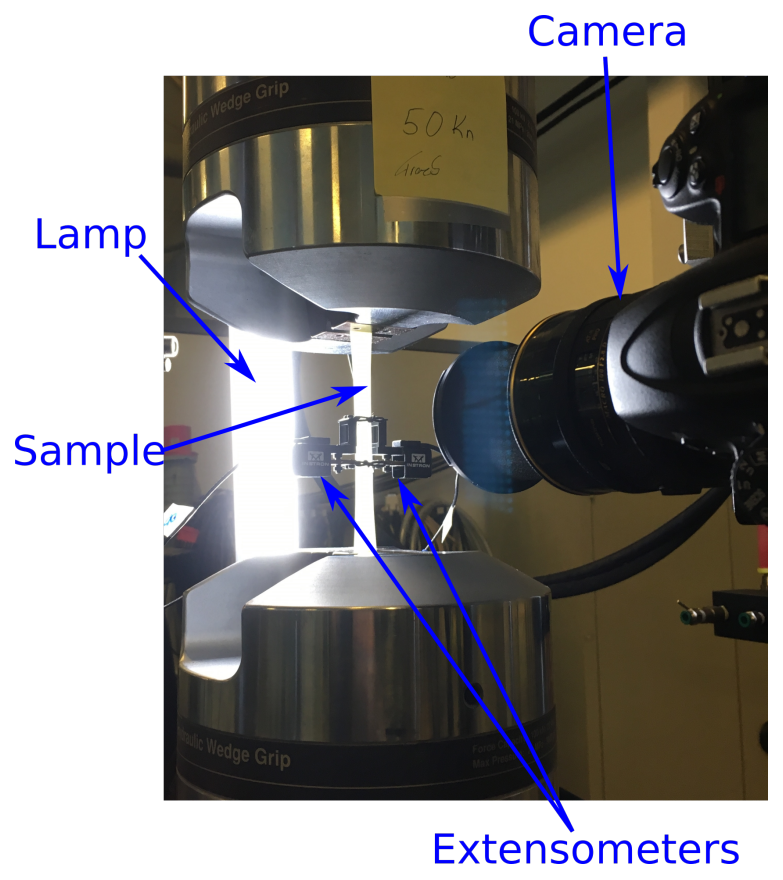

Figure 5: Fatigue testing setup with camera in front of the sample and illuminating LED panel on the back.

The specimen EPS06-1 (see Table 2) was used for the ex-situ experiments. The ex-situ X-ray CT fatigue test was interrupted after 100,000, 150,000, 200,000, and 
500,000 cycles for X-ray CT examination. The specimen was also initially scanned at high resolution before any load was applied. The specimens EPS06-3 and EPS06-4 were used for the tension clamp experiments and were interrupted after 100,000 and 1,000,000 cycles, respectively.

\subsection{X-ray Computed Tomography}

All the X-ray CT experiments were carried out on a Zeiss Xradia Versa 520 scanner at an accelerating voltage of $80 \mathrm{keV}$ and a power of $7 \mathrm{~mA}$. A 2000x2000 pixel detector was used with a binning of 2 was used resulting in 1000x1000 pixels in the final projection images.

The X-ray CT scans carried out to obtain the backing fibre bundle structure were performed with an optical magnification of $0.4 \mathrm{x}$. . To obtain a field of view of around 20x20mm (covering the region considered by the TWLI experiments) the scans were performed with a source-to-sample distance of 40mm and a detector-to-sample distance of $100 \mathrm{~mm}$ resulting in a voxel size of $19.5 \mu \mathrm{m}$. The scan time for each scan was a bit less than 2 hours.

For the ex-situ experiments an optical magnification of $4 \mathrm{x}$ was used and the sourceto-sample distance was $20 \mathrm{~mm}$ and a sample-to-detector distance $40 \mathrm{~mm}$ giving a pixel size of $2.32 \mu \mathrm{m}$ in the projection images. The projection images were captured with an exposure time of 2.5 seconds leading to a scan time of more than 5 hours. The tension clamp experiments were carried out with similar settings, but because of the presence of the clamp it was necessary to increase the source-to-sample distance to $25 \mathrm{~mm}$ and the sample-to-detector distance to $50 \mathrm{~mm}$ to obtain a similar pixel size $(2.25 \mu \mathrm{m})$. This resulted in an exposure time of 5 seconds for these scans and a scan time of around 10 hours. To obtain good image quality the performed scans were performed with 5201 projections.

Image reconstruction was performed using the "XMReconstructor - Cone Beam 10" from Zeiss, and data visualisation was done using the open source software "ImageJ" [37]. All the reconstructed X-ray CT data sets can be downloaded online [36]. Each 
reconstructed dataset is $2 \mathrm{~GB}$ in size. For the ex-situ experiments there are 8 data sets and for the tension clamp experiments there are 4 data sets.

\subsection{Tension clamp experiments}

A tension clamp solution was used to apply load to the specimen during X-ray CT examination of the specimens EPS06-3 and EPS06-4 interrupted after 100,000 and 1,000,000 cycles, respectively. Hence, the tension clamp experiments both considered fatigue damage of specimens relatively early and late in the fatigue life as also illustrated earlier in Fig. 1. The tension clamp was a modified version of the clamp described in [19] that can apply static load to the specimen during the X-ray CT experiments and is also explained in more detail in Appendix A.

In the current study, the specimen was initially loaded up just below $0.6 \%$ strain, and the strain applied by the clamp was measured to be around $0.5 \%$ after leaving it for around one hour after it was attached. At this point the strain seemed to have stabilised, but as will be clear from the results presented later, some relaxation occurred during the subsequent scanning.

\section{Crack quantification method}

The crack detection algorithm used for automatically quantifying the damage is explained in detail in [35] and includes three overall steps; image compensation, image filtering, and crack counting briefly outlined in Appendix B.1, which also explains some necessary modifications to the original method.

One challenge when counting cracks both automatically and manually is to validate the results. In the current study, the crack counting for all the tests were performed with the same input parameters for the counting algorithm. This means that cracks with sufficient contrast in the images will be counted similarly for all the tests, and thereby be comparable to one another. In addition, manual inspection of the counted cracks relative to the actual cracks in the images show good agreement between the counted and the visible cracks, as can also be seen from Fig. 6. 


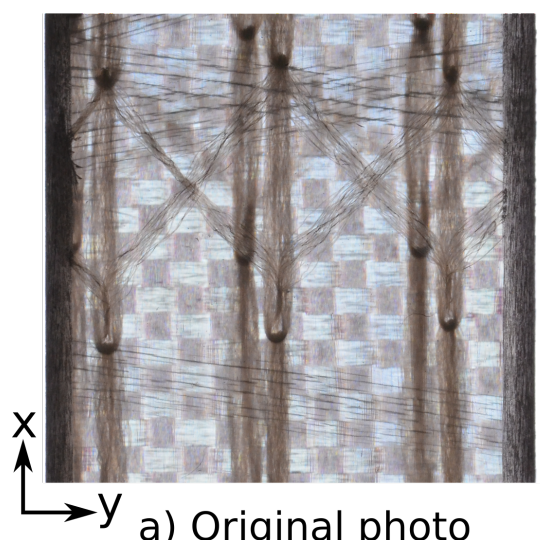

a) Original photo

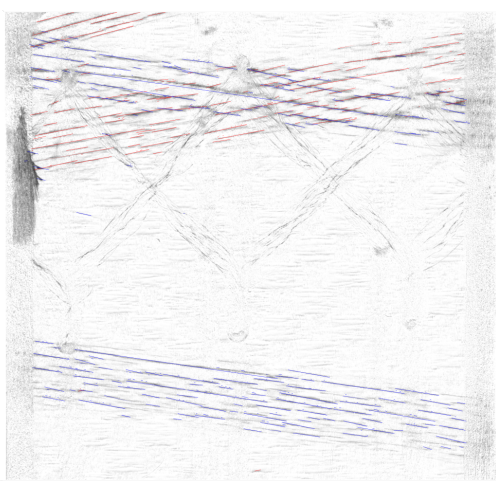

b) Counted cracks

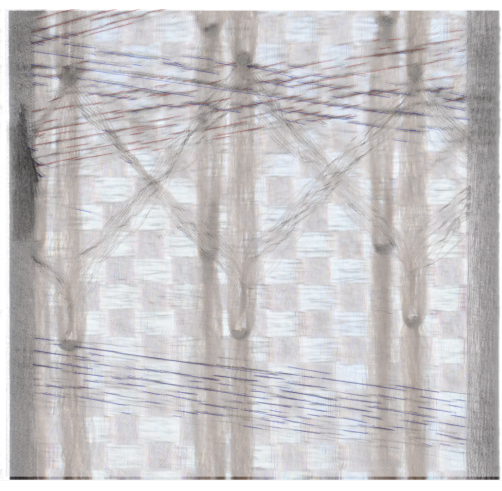

c) Cracks overlayed on original photo

Figure 6: Example of counted cracks compared to the original photo for EPS06-1

\subsection{Crack density relative to bundle structure}

For composites with a reasonably homogeneous distribution of fibres within each layer, the crack density is usually defined proportional to the total surface area of the considered region of specimen as done in [6, 35]. However, the considered composite has a coarse fibre bundle structure with large regions of pure matrix. Therefore, it is necessary to define the off-axis crack density relative to the backing fibre bundle area to make them comparable to one another. In the present work, the crack density relative to the bundle structure, $\rho$, is defined as:

$$
\rho=\frac{\sum_{i=1}^{N_{c}} L_{i}}{A_{b b}}
$$

where $\sum_{i=1}^{N_{c}} L_{i}$ is the sum of the measured length of all observed cracks, and $A_{b b}$ is the 'projected backing bundle area', which corresponds to the 2D surface area of all the backing bundles when looking down on the x-y plane defined in Fig. 3a (see also Fig. B.2 in Appendix B.2.

In order to obtain the backing bundle structure of the considered region, a large field of view X-ray CT scan was performed on all the tested samples prior to fatigue testing, as mentioned earlier in Section 2.3. In principle, it would be possible to extract the bundle structure in 3D from the X-ray CT images. However, at present there is no 
automated way to do so and therefore it would require complete manual segmentation of the bundles. This is time consuming, and with the relatively large voxel size of 19.5 microns it is difficult to distinguish the backing fibre bundles from the UD fibre bundles at locations where they lie close to one another. Therefore, a simple method for extracting the projected backing bundle area in 2D was established as explained in more detail in Appendix B.2.

\section{Off-axis cracks and the effect of strain level}

The off-axis crack initiation and progression is a complex process and will therefore be discussed first based on qualitative observations. This is then followed by discussions based on quantitative measures of the crack density. Here the effect of the applied initial peak strain of the fatigue tests on the initiation and progression of the off-axis cracks is also discussed.

\subsection{Crack initiation and growth behaviour of off-axis cracks}

Fig. 7 shows an example of the cracks in the EPS08-1 sample (see Table 2 ) after 100,000 cycles (Fig. 7a) along with the same photo with the projected backing bundle

area shown on top (Fig. 7b). The projected backing bundle area and the cracks have been manually aligned to obtain Fig. 7b. Although the backing fibre bundles cannot be seen in the camera images, Fig. 7 shows that the pattern in which the cracks appear match the projected backing bundle area obtained by X-ray CT. Hence, the visible off-axis cracks are not evenly distributed in the sample, but appear nearly exclusively in the backing fibre bundles. This is the case for all the tests. It should be noted that the cracks monitored by TWLI are cracks in the backing layer located in the centre of the composite, hence not surface cracks.

Although the lay-up definition of the fabrics refers to the backing as $\pm 80^{\circ}$, there is a significant variation in the backing fibre bundle structure from sample to sample and even within each individual sample (see also Fig. B.4 in Appendix B.2). This variation 


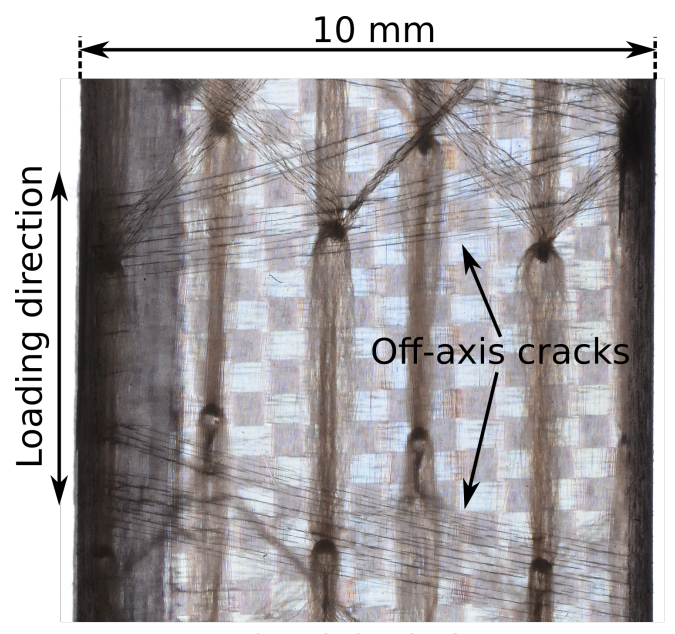

a) Original photo

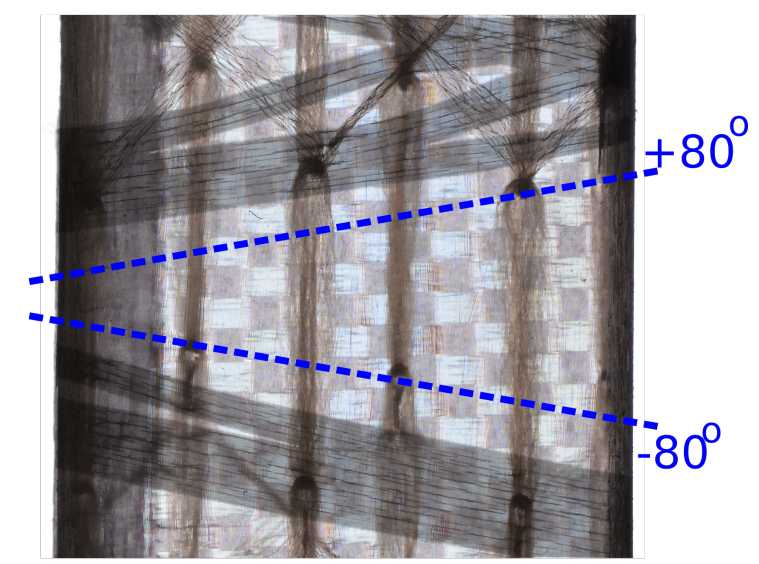

b) Photo with projected backing bundle area

Figure 7: Example of (a) cracks in the backing bundles for sample tested at $0.8 \%$ strain for 100,000 cycles and (b) the same photo overlayed with the backing bundles obtained by X-ray CT marked in grey on top.

seems to be a result of the stitching procedure that locally influences the backing fibre bundle orientation during manufacturing of the fabric.

Fig. 8 shows an example of the off-axis crack progression for a region in one of the samples tested at a peak strain of $\epsilon_{\max }=0.6 \%$ (EPS06-2). Videos showing the off-axis crack progression for all the performed tests can be found online [36]. Fig. 8a shows the sample before any load was applied, and no off-axis cracks were present at this stage. For this strain level, $\epsilon_{\max }=0.6 \%$, some off-axis cracks already initiated after the first 
cycle as shown in Fig. 8b. This was the case for all the tests performed at strain levels of $0.6 \%$ and $0.8 \%$. With further load cycles the number of off-axis cracks increased, as seen by comparing Fig. 8b, c, and d. In general, off-axis cracks were observed to initiate at different times and grow in parallel to one another.

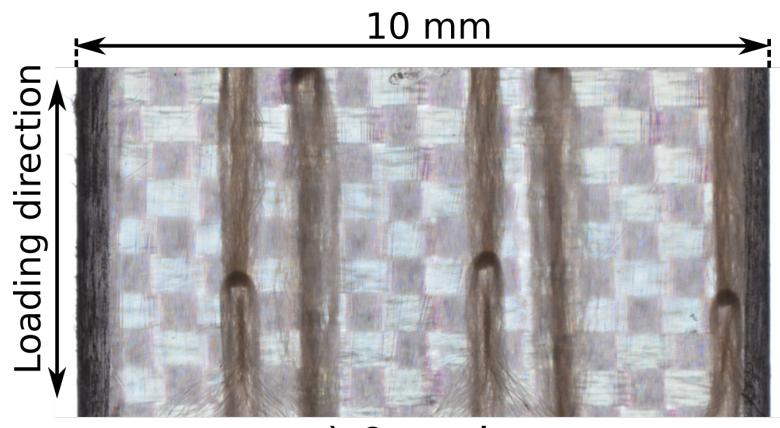

a) 0 cycles

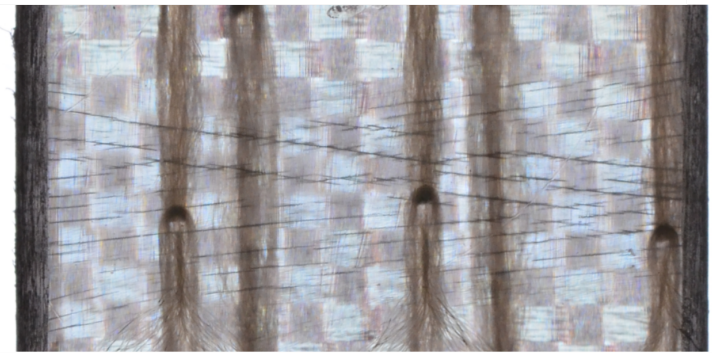

c) 1000 cycles

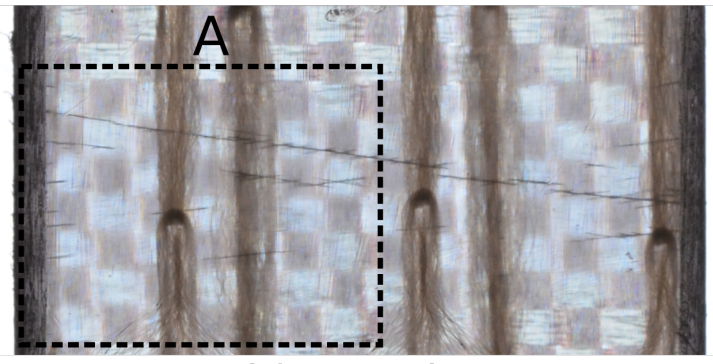

b) 1 cycle

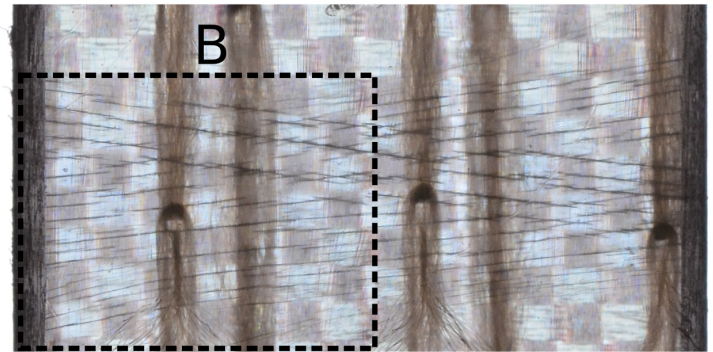

d) 100000 cycles

Figure 8: Example of off-axis crack growth at $0.6 \%$ strain (Eps06-4). Zoom views of the two marked regions $\mathrm{A}$ and $\mathrm{B}$ are shown in Fig. 9

Fig. 9 shows zoom views of the regions A and B marked in Fig. 8. From Fig. 9a it is seen that the cracks both initiate from the edges as well as inside the specimen. Fig. 9a also shows small $-80^{\circ}$ cracks initiating as small "branches" from a $+80^{\circ}$ crack at the cross-over region of the backing fibre bundles. Looking at Fig. $9 \mathrm{~b}$ it can also be seen that some cracks appear wider than others. Similar effects were observed for all the tests (e.g. see videos online [36]).

\subsection{Effect of strain level on the off-axis crack development}

Fig. 10a shows a semi-logarithmic plot of the crack density measured using the method described in Section 3 and Appendix B.1 as a function of the fatigue load 


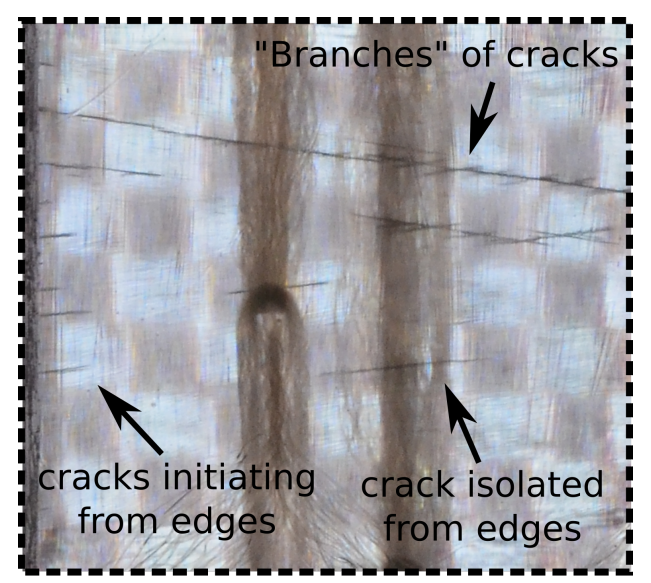

a) Zoom view $A$

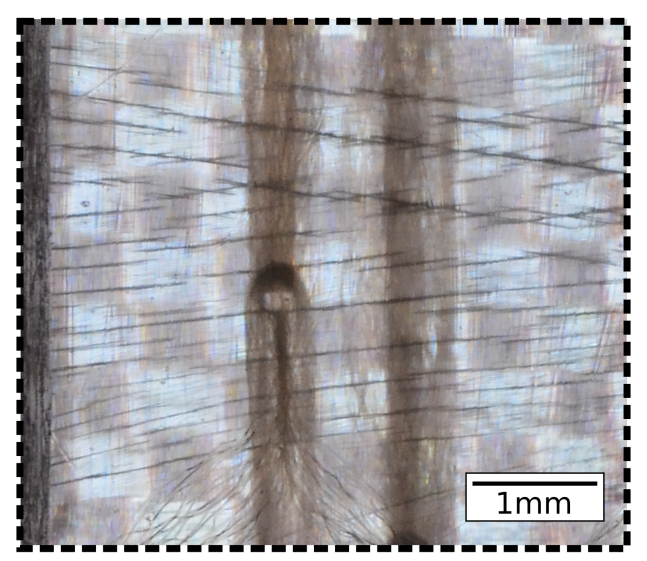

b) Zoom view B

Figure 9: Selected zoom views from Fig. 8 (the same region at two different damage states)

cycles for all the samples. For all the tests performed at $0.8 \%$ and $0.6 \%$ strain, one of the tests at $0.5 \%$ and one of the tests at $0.4 \%$ strain, it is seen that cracks already have formed after the first cycle. There seems to be a high degree of scatter in the static strain threshold limit for the initiation of off-axis cracks, which is likely to be related to the variation in the fibre bundle architecture.

\subsubsection{Crack interaction and saturation}

Although no clear saturation crack density can be determined from the semi-log plot in Fig. 10a, it can be seen more clearly from Fig. 10b that the curves are starting to level out going towards an apparent saturation average crack spacing (and crack density). The applied maximum strain seems to affect the apparent saturation crack 


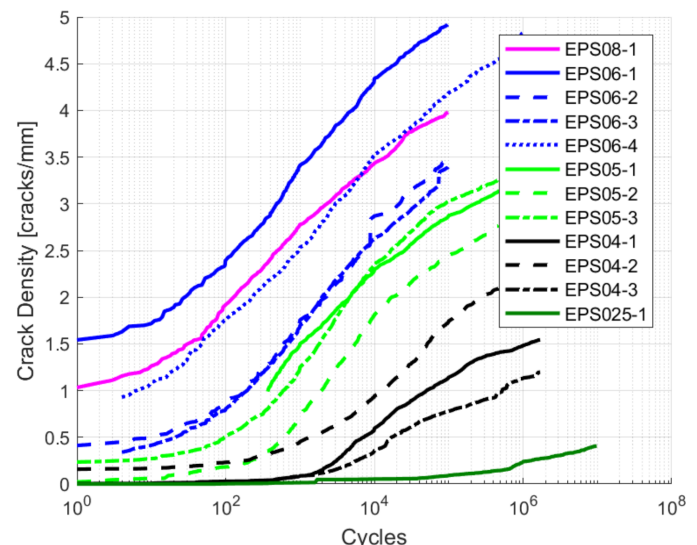

a) Off-axis crack density vs log(cycles)

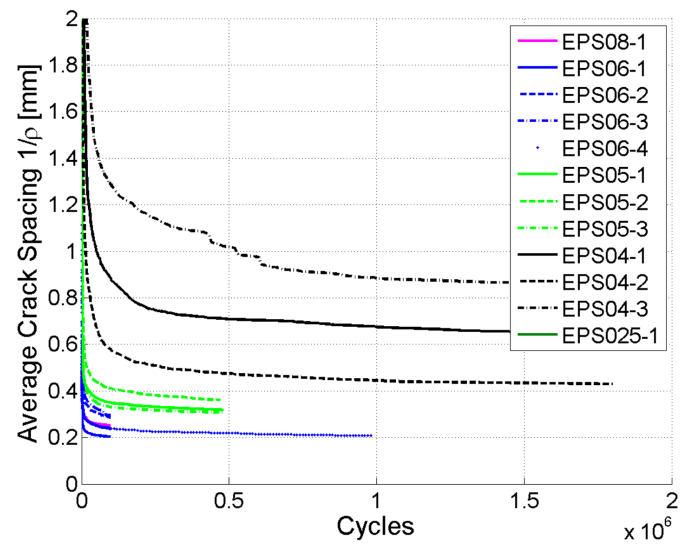

b) Average crack spacing vs cycles

Figure 10: Plots of a) the off-axis crack density as a function of the fatigue load cycles for all the performed tests (note the plot is semilogarithmic) and b) the average crack spacing $(S=1 / \rho)$ as a function of the number of fatigue load cycles.

density, hence the saturated number of off-axis cracks in the backing fibre bundles will be different depending on the strain level chosen for the fatigue test. As will be discussed also later, the off-axis cracks in the backing fibre bundles are highly related to the initiation and growth of more critical damage such as UD fibre fractures. Therefore, accelerating a fatigue test by testing at higher strain levels than those present in the actual structure might affect how the damage evolves. Similar observations have also been reported for other lay-ups e.g. [6, 38]. However, the case of $0.8 \%$ strain is very similar to the $0.6 \%$ strain tests. It could be that above a certain strain level the growth behaviour of the off-axis cracks is less affected by the strain level, however this would need further investigation.

Fig. 10b shows the development of the average crack spacing, $S=1 / \rho$, inside the backing bundles as a function of cycles for the tests. The average crack spacing obtained when each of the tests were stopped was $0.25 \mathrm{~mm}$ for $0.8 \%$ strain, in the range 0.20 $0.29 \mathrm{~mm}$ for $0.6 \%$ strain, $0.31-0.36 \mathrm{~mm}$ for $0.5 \%$ strain and $0.43-0.83 \mathrm{~mm}$ for $0.4 \%$ strain. For $0.25 \%$ strain the crack density was still so low that the average crack spacing was unrealistically high and was therefore left out. The backing fibre bundle thickness was measured approximately in the X-ray CT images, and was found to go down to $0.07 \mathrm{~mm}$ 
for single bundles and up to $0.25 \mathrm{~mm}$ for cross-over regions. Crack interaction typically occur when the crack spacing is comparable to the layer thickness (S smaller than five times the layer thickness [38]), and for a quasi-isotropic laminate subjected to fatigue loading Tong 38] observed crack saturation when the crack spacing was around the same as the layer thickness. From Fig. 10b it is seen that particularly for $0.6 \%$ strain the final crack spacing is similar to approximate the backing fibre bundle thickness. However, for the lower strain levels the apparent saturation seems to be obtained at a significantly larger value of the crack spacing.

\subsection{Applying the method to compare material systems}

Due to the long fatigue life-time $\left(10^{8}-10^{9}\right.$ cycles $)$ the tests performed for the UD NCF composites used for wind turbine blades are usually accelerated by testing at a higher strain. However, the results presented through Section 4 indicate that accelerating the test by testing at higher strain levels influences the off-axis crack saturation density and thereby likely also subsequent damage in the material. The automatic crack counting method presented above makes it possible to compare the fatigue properties of material systems at realistic strain levels. This could be done by establishing an S-N curve for different crack densities as shown in Fig. 11 for the material considered in the current study. The curve shows how many cycles it took to reach a certain crack density, in this case $\rho=1,2,3$. The test performed at $0.25 \%$ strain only reached a crack density of 0.19 after $10^{7}$ cycles and therefore is not part of Fig. 11. The lines shown in Fig. 11 are manually drawn trendlines. Such an approach provides a way to describe the damage state of the composite aside from measuring the stiffness degradation, which does not solely describe one damage mechanism.

As the fatigue damage progression is highly related to the off-axis cracks in the backing fibre bundles, the type of curve shown in Fig. 11 gives a simple method to compare the fatigue properties of quasi-UD NCF composites. For example it can be used to compare the effect of various manufacturing conditions such as the curing temperature and curing cycles, different constituent materials, fibre diameters or similar. 


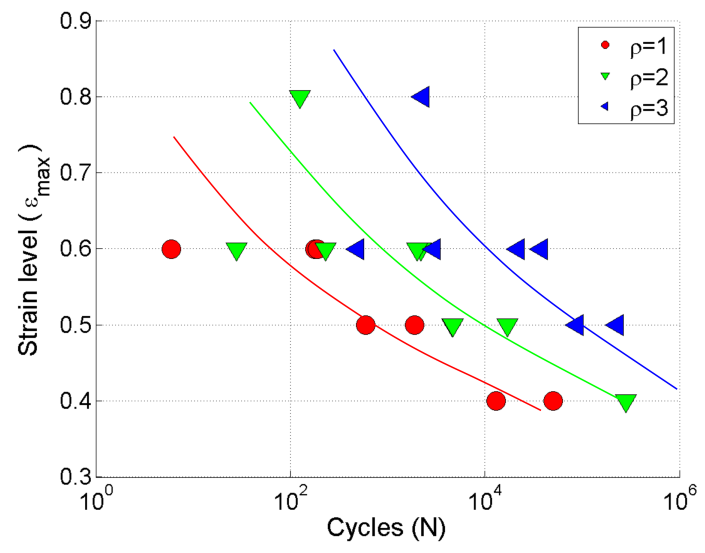

Figure 11: S-N curve for different crack densities with emprical trendlines.

In addition, linking the off-axis crack density with the subsequently occurring critical damage mechanisms such as UD fibre fractures could make it possible to predict the lifetime based on the initial damage states in the future. Monitoring the progression of UD fibre fractures to get closer to such an understanding is considered in the next section.

\section{UD fibre fractures}

In this section, the results obtained from the ex-situ fatigue study and the tension clamp experiments will be presented and discussed. Fig. 12 shows the stiffness degradation measured during the fatigue tests of the three considered specimens EPS06-1, EPS06-3, and EPS06-4. For EPS06-1 subsequently tested further during the ex-situ test, the data is only included until the first interruption point. Dismounting and remounting the extensometers seemed to have affected the measured stiffness degradation significantly for the test, and therefore this data is not included. Nevertheless, the measured stiffness degradation for the tests is seen to have a shape similar to that shown earlier in Fig. 1. Furthermore, assuming they will follow a similar trend to EPS06-4, the interruption points for EPS06-1 and EPS06-3 are seen to be around where the curve begins to show a linear degradation behaviour, hence in the end of stage I. EPS06-4 is seen to have been interrupted significantly later in stage II the fatigue life. 


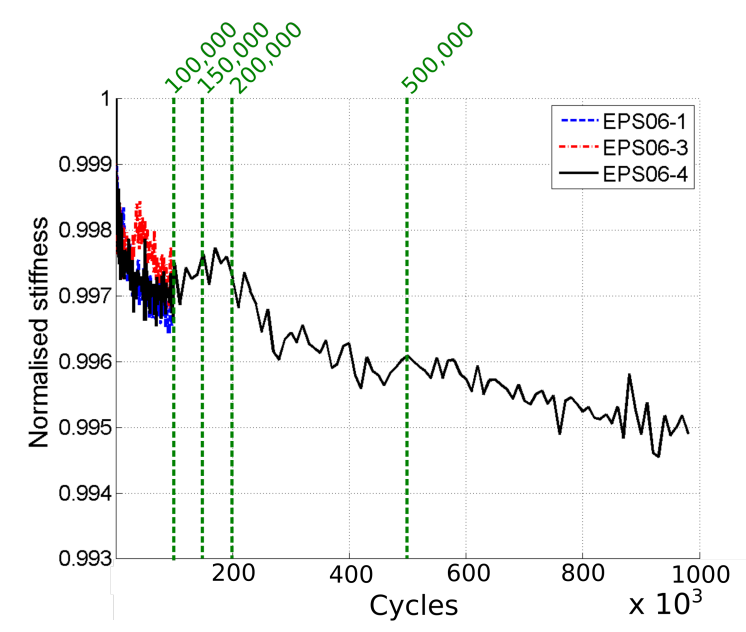

Figure 12: Stiffness degradation of the considered tests

\subsection{Initiation and progression of UD fibre fractures}

In a previous study considering a rather similar material system [18], UD fibre fractures were found to initiate primarily at locations where the backing fibre bundles were crossing over one another and at the same time were in 'contact' with a UD fibre bundle. Here being in 'contact' means that the bundles lie so close to each other that the distance between their fibres become similar to that inside the individual bundles. In the current study this information was used to determine the locations to scan, where damage was expected to initiate. Performing a large field of view scan before fatigue testing made it possible to zoom in a region chosen based on the bundle structure. This was done using the Scout-and-Scan principle provided by Zeiss. Ex-situ experiments were carried out in two regions: one including a cross-over region of the backing in contact with the UD bundles (region I) and another where a single backing fibre bundle is touching a UD bundle only on one side (region II). The full 3D data sets can be found online [36].

Fig. 13 shows a 2D slice view of the damage progression observed in region I by ex-situ X-ray CT. Before any load was applied, no damage could be observed in the X-ray CT images. At the first interruption point after 100,000 cycles, a few UD fibre fractures was seen to have initiated at the cross-over region as also marked by the dashed 
line marking in Fig. 13b. UD fibre fractures were seen to gradually progress from the cross-over region of the backing fibre bundles in contact with the UD fibre bundle and into the thickness direction of the UD bundle. This is seen by the difference between Fig. 13b and 13k. After 500,000 cycles the UD fibre fractures had progressed even further and already existing fibre fractures had become more visible. This indicates an increased crack face opening of the fibre fractures, even though it was scanned by X-ray $\mathrm{CT}$ in the unloaded state. This kind of permanent increase of the crack face opening of fibre fractures in the unloaded state have also been observed in a previous study for a different UD NCF composite [18].

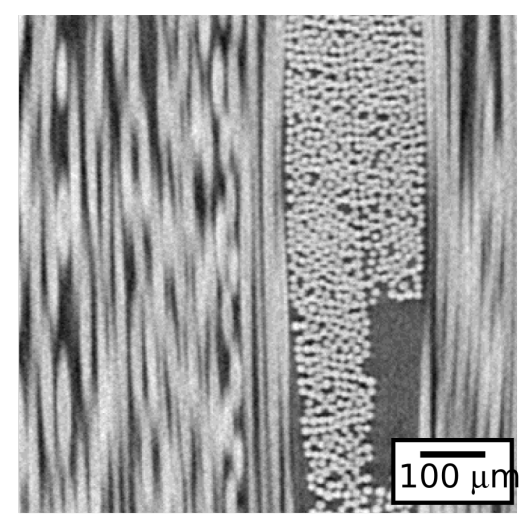

a) $\mathrm{N}_{0}=0$

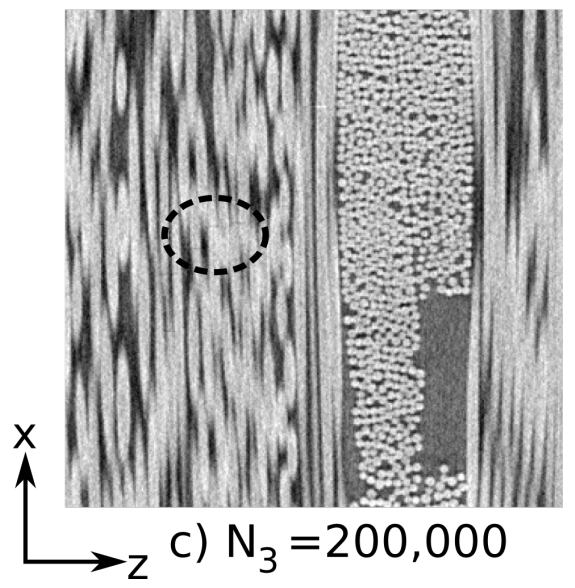

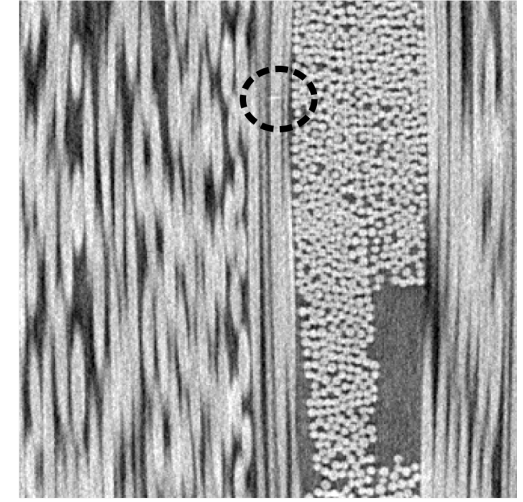

b) $\mathrm{N}_{1}=100,000$

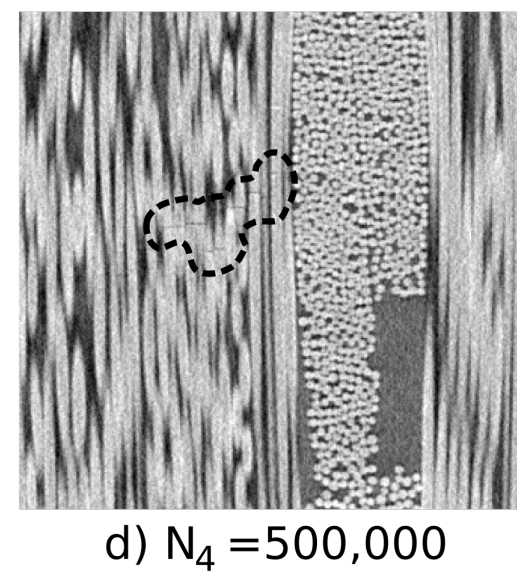

Figure 13: A 2D slice view in Region I in specimen EPS06-1 during the ex-situ test. The markings with dashed lines show regions with UD fibre fractures that have become visible.

Another thing that can be observed from Fig. 13, is that off-axis cracks in the 
backing fibre bundles are not visible. However, Fig. 14 shows photos of the off-axis cracks near scan region I captured by TWLI, and it is seen that several off-axis cracks are present already at the first interruption point of the ex-situ study. Hence, the resolution in the X-ray CT images is not sufficient to see the off-axis cracks in the backing fibre bundles, since the off-axis cracks are practically closed. Previous studies [18, 19] have also shown the difficulty of seeing this type of cracks by X-ray CT, which is also the reason why the current study included TWLI in the ex-situ fatigue testing approach. Fig. 14 also shows that there is no significant change in the off-axis crack density after the first interruption point of the ex-situ test, which indicate that they have reached saturation as was also argued in Section 4.

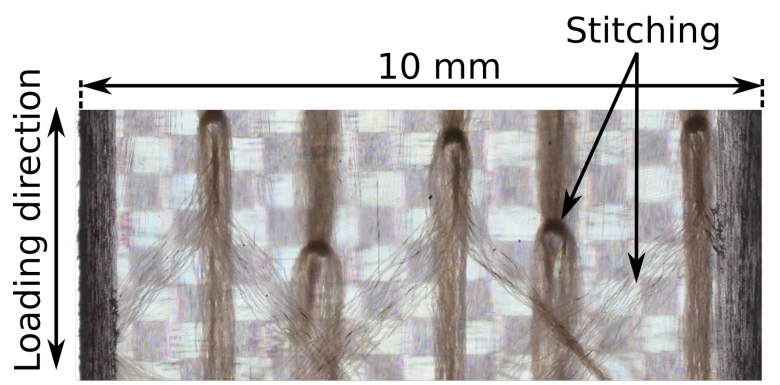

a) 0 cycles

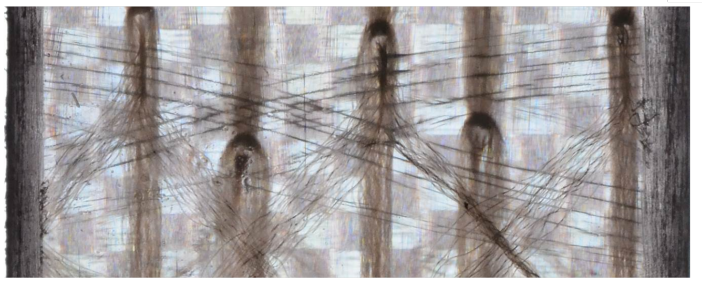

c) 200,000 cycles

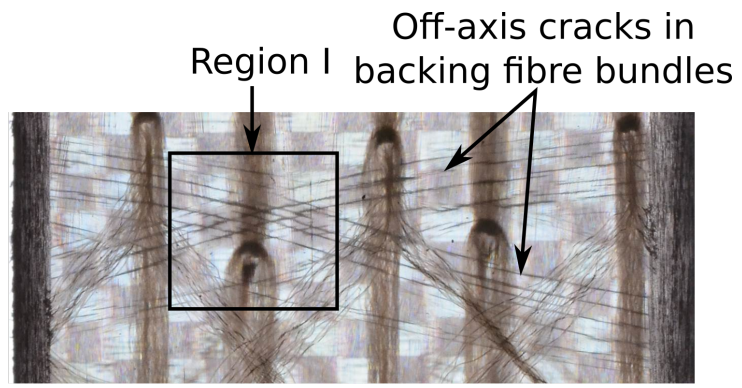

b) 100,000 cycles

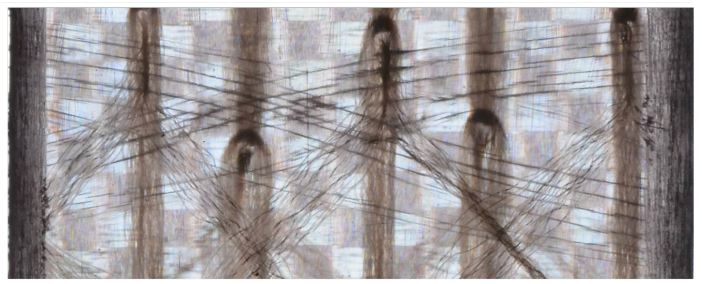

d) 500,000 cycles

Figure 14: TWLI images showing off-axis cracks in the backing fibre bundles near region I of specimen EPS06-1 at each of the stopping points for the ex-situ test shown in Fig. 13 The checkerboard pattern visible in the images is the peel-ply on the specimens.

Although region II did not include a cross-over region, UD fibre fractures could still be observed in the UD fibre bundle in contact with the backing fibre bundle. Nevertheless, to a smaller extent than for region II making it difficult to see in 2D slice views. UD fibre fractures were observed to appear near the cross-over region (region 
I) earlier in the fatigue life than near the single backing fibre bundle (region II). In addition, the damage region near the cross-over region appeared larger than at the single backing fibre bundle region. For an example of the observed fibre fractures in region II, the reader is referred to [36] where the raw 3D data also can be found.

\subsection{Effect of applied tension on the crack visibility}

One thing that is clear from the damage progression presented in the previous section, is that the crack face opening of the fibre fractures observed in the images is relatively small. It is likely that additional fibre fractures with no or openings smaller than the resolution exist but cannot be seen in these images. Therefore, load was applied during X-ray CT scanning to open up cracks and study the effect of applied load on the crack visibility in the X-ray CT scans. Two samples (EPS06-3 and EPS064) were scanned in two different regions each, with and without tension clamp applied. The specimens were scanned with load applied first and then subsequently scanned in the unloaded state. Therefore, even if not visible, any damage present in the loaded state also exists in the unloaded state. Fig. 15 shows the locations of the scan regions on top of the projected backing bundle area (region A-D). The full 3D data sets can be found online [36]. The off-axis cracks observed by TWLI are shown for each region in the figure. From the TWLI images it is seen that several off-axis cracks were present in all the considered regions when the X-ray CT scans were performed.

Fig. 16 shows 2D slice views from the 3D images of the two regions examined by X-ray CT with and without applied tension during scanning in the specimen EPS06-3 (region A and B). The fatigue test of specimen EPS06-3 was interrupted after 100,000 cycles, which is relatively early in the fatigue life (around the end of stage I). Fig. 163 shows a region with a single backing fibre bundle (region A), and Fig. 16b a crossover region of the backing fibre bundles (region B). A significant improvement of the visibility of cracks can be observed when tension is applied during X-ray CT scanning. As seen in Fig. 16a, no off-axis cracks nor fibre fractures were visible in region $\mathrm{A}$ in the unloaded state. However, when load was applied, several UD fibre fractures became 


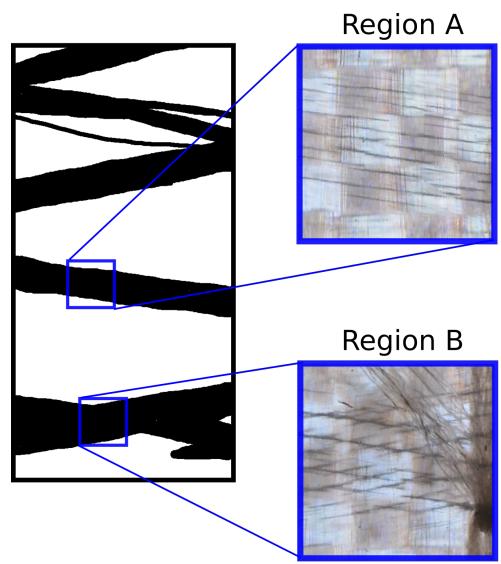

a) EPS06-3

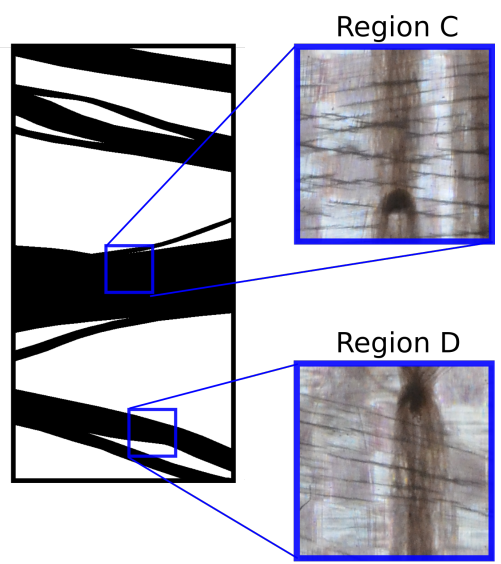

b) EPS06-4

Figure 15: Locations of the scan regions on top of the backing fibre bundle structure for the tension clamp experiments for (a) the specimen EPS06-3, and (b) the specimen EPS06-4. The TWLI images of the off-axis cracks are shown for the four considered regions (A-D).

visible and some off-axis cracks could also be seen in the backing fibre bundles near the UD fibre fractures.

For region B shown in Fig. 16b, it is seen that the tension clamp had caused the X-ray CT image to be out of focus. The images look similar to a reconstruction with incorrect centering of the image, but it was caused by movement during scanning. Region B was scanned prior to region A and the load applied by the clamp must have relaxed a bit during the first scan. However, since this problem was not observed for the subsequent scan of region A, it must mean that the relaxation leveled out after several hours (each X-ray CT scan took around 10 hours). Hence, it is necessary to wait longer than one hour before X-ray CT scanning of a sample with the tension clamp applied to avoid the relaxation to affect the image quality. Nevertheless, despite the blurry image it is clear that there is a significant number of UD fibre fractures that were not visible in the unloaded state.

Fig. 17 shows $2 \mathrm{D}$ slice views from the $3 \mathrm{D}$ images of the two regions examined by X-ray CT with and without applied tension during scanning in the specimen EPS06-4. This test was interrupted after 1,000,000 cycles, which is relatively late in the fatigue 


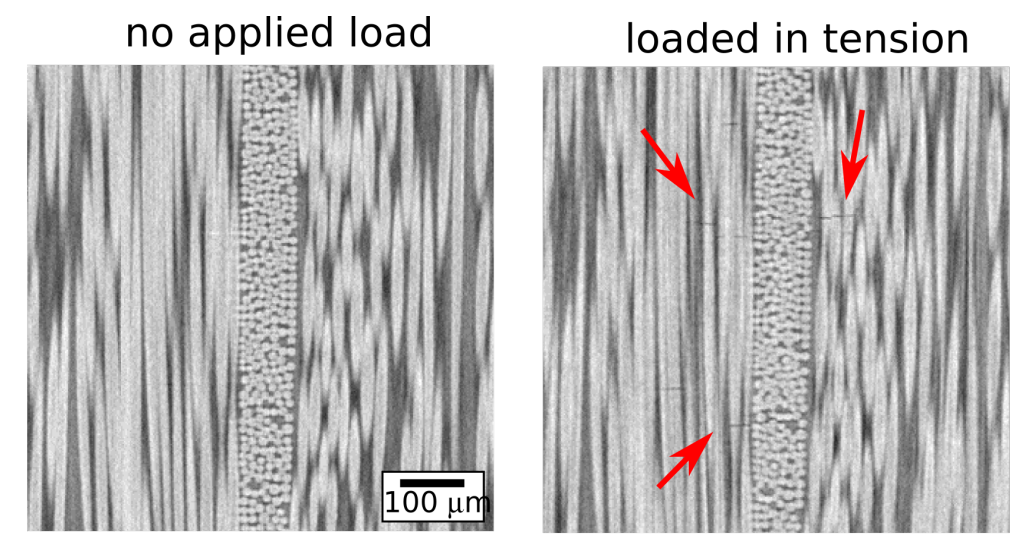

\section{a) Single backing bundle (region $A$ )}

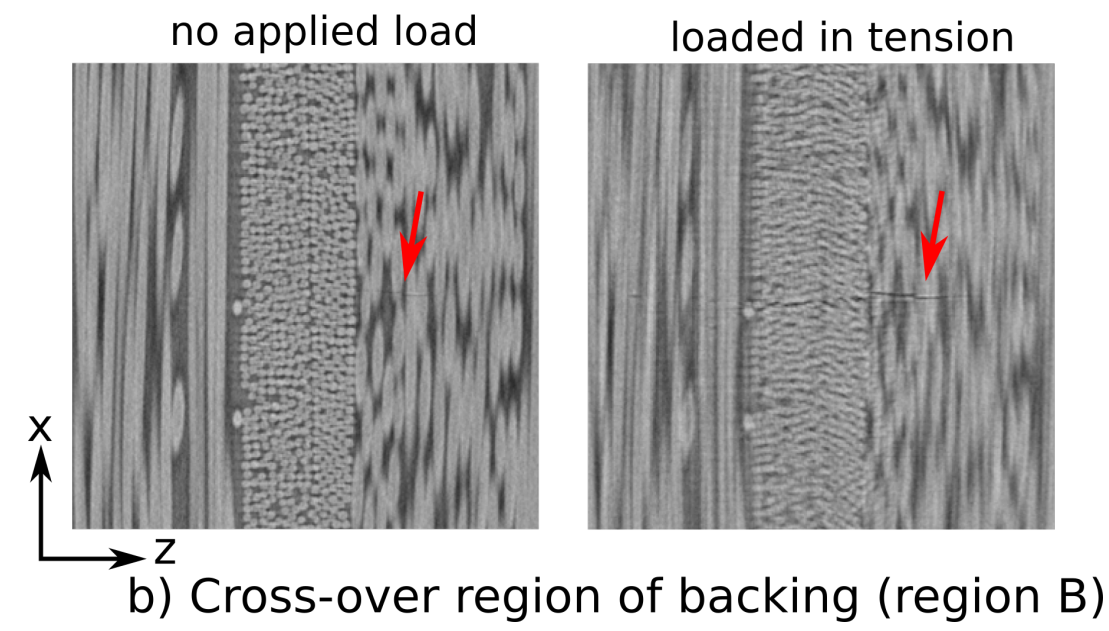

Figure 16: 2D slice views of region A and B (see Fig. 15) for EPS06-3 (100,000 cycles) with and without applied load during X-ray CT scanning.

life (see also Fig. 12). Fig. 17b shows a backing fibre bundle, which is in contact with only one UD bundle. As was also seen for the case shown in Fig. 16, the load has affected both the visibility of the UD fibre fractures and the off-axis cracks significantly. Furthermore, the tension clamp experiments showed that UD fibre fractures also exist at single fibre bundles, which was not observed in previous X-ray CT studies on this particular quasi-UD composite [18, 20].

\subsection{Off-axis cracks as damage initiators}

In addition to making it easier to see the UD fibre fractures, applying tension during the X-ray CT scan also increased the visibility of the off-axis cracks in the backing fibre 


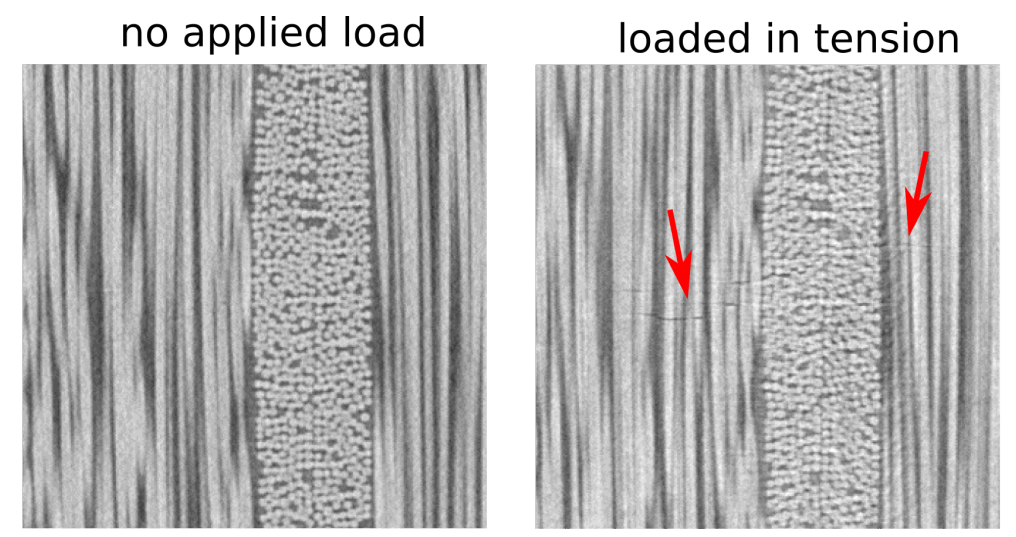

a) Cross-over region of backing (region C)

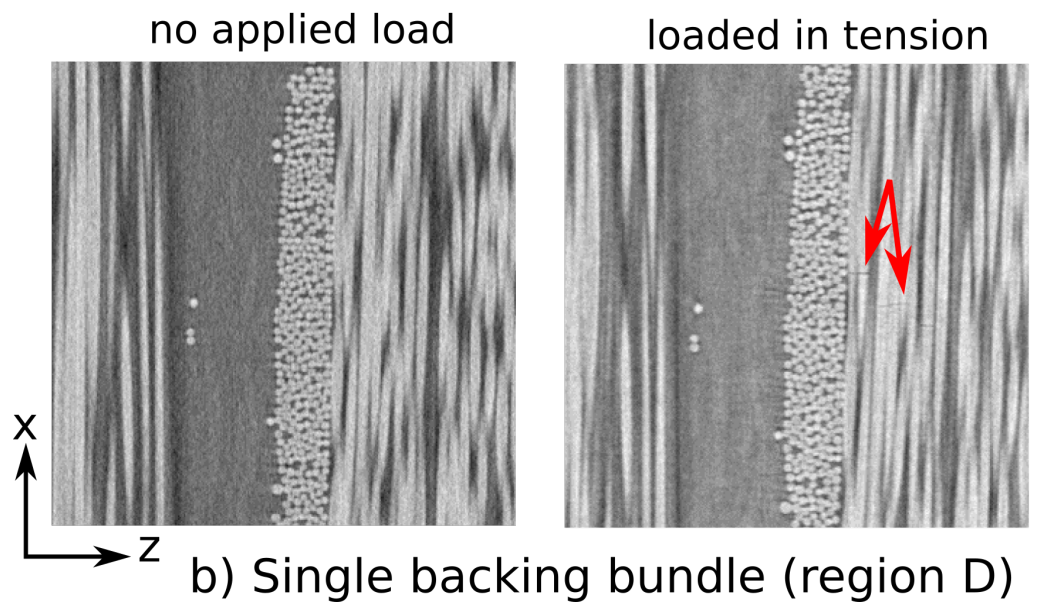

Figure 17: 2D slice views of region C and D (see Fig. 15) for EPS06-4 (1,000,000 cycles) with and without applied load during X-ray CT scanning.

bundles as seen from the examples in Fig. 18. The transilluminated white light imaging experiments performed, showed that off-axis cracks are present even if not visible in the X-ray CT scans. When the tension clamp was applied, it was possible to see indications of these off-axis cracks at the regions with UD fibre fractures as also seen from Fig. 18. These observations strongly indicate that the UD fibre fractures indeed have initiated from off-axis cracks in the backing fibre bundles, as also argued in previous studies [17, 19]. Furthermore, even with load applied during X-ray CT scanning, UD fibre fractures were not observed when there was a layer of matrix of a certain thickness in between the backing fibre bundle and the UD fibre bundle. This is believed to be 
because UD fibre fractures initiate from stress concentrations at the tips of the off-axis cracks in the backing, and that a sufficiently thick layer of matrix causes the crack to arrest.

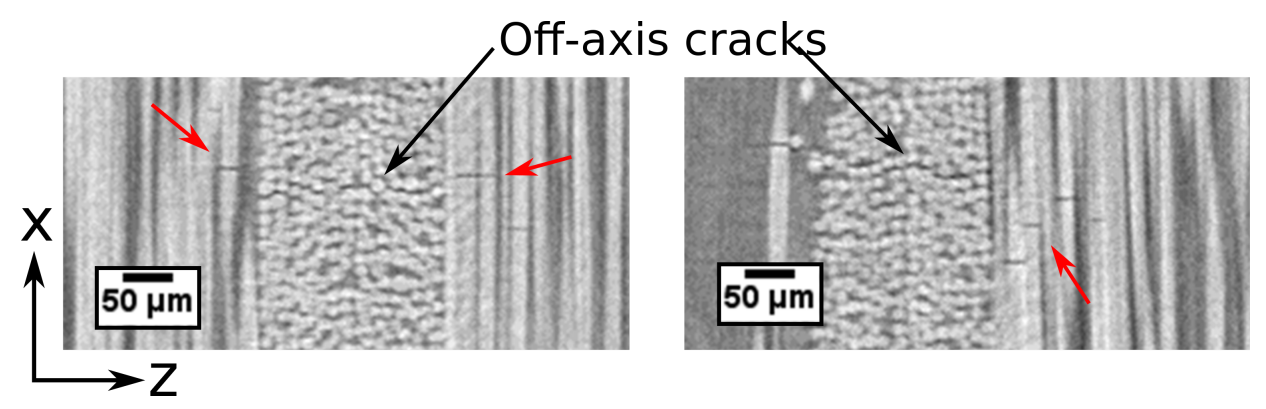

Figure 18: Examples of off-axis cracks visible for EPS06-04 with the tension applied during CT scanning using the tension clamp.

\subsection{UD fibre fractures during the early fatigue life}

From the tension clamp experiments shown in Fig. 16 it was seen that a significant number of UD fibre fractures were present even after 100,000 cycles, which is around the end of stage I of the fatigue life. In addition, studies on prepreg based laminates often considered in the aerospace and automotive industries also state that fibre fractures occur over all stages of the fatigue life [9], and in this regard it is likely that similar mechanisms come into play for the considered quasi-UD composites.

Although present, fibre fractures are generally disregarded in prediction models, since they are not the controlling damage mechanism of stage I and II of the fatigue life for common layups such as quasi-isotropic and cross-ply laminates. In the case of composites with thicker off-axis layers, the fibre fractures appearing early in the fatigue life might have a smaller contribution to the normalised stiffness degradation of the composite, since the off-axis layers contribute to a significantly larger percentage of the total composite stiffness than for the considered quasi-UD composites. In the case of quasi-UD composites, the initiation of a significant number of fibre fractures might overshadow the stiffness degradation from off-axis cracks in the backing fibre bundles, 
since the backing fibre bundles contribute only a few percent to the axial stiffness of the composite, and is therefore important to take into account.

\section{Modified fatigue damage progression scheme}

In a previous study by Zangenberg et al. [17] a damage progression scheme for a quasi-UD composite similar to the one considered in the current study was presented. The damage progression scheme was established based on scanning electron microscopy images at one damage state late in the fatigue life. Therefore, the order in which the damage mechanisms appeared could only be based on speculations. Using the additional information obtained through the current study, the damage progression scheme by Zangenberg et al. [17] has been slightly modified and expanded. Fig. 19 shows the modified version of the damage progression scheme, where the initial state with no damage has been left out. The graph in the center of Fig. 19 shows the typical stiffness degradation of a fibre composite with five points marked (a-e). The figure include a principle sketch of the damage present at each of the damage states associated to these points.

The main modification done to the damage progression scheme is in the early fatigue life. The original scheme stated that off-axis cracks in the backing fibre bundles would initiate first in all the backing fibre bundles, and that this was the cause of the rapid stiffness degradation observed during stage I of the fatigue life. Although the current study showed that the off-axis cracks indeed initiate early in the fatigue life, the results also showed that UD fibre fractures were present during stage I. Therefore, it is believed that some UD fibre fractures initiate together with the off-axis cracks in the backing fibre bundles as illustrated in Fig. [19a.

The TWLI experiments showed that the off-axis cracks might not initiate simultaneously in the different backing fibre bundles. This is shown in the scheme by the difference between Fig. $19 \mathrm{a}$ and $19 \mathrm{~b}$. In addition, new off-axis cracks might initiate between existing cracks in the backing bundles as shown in the bottom-left backing

fibre bundle shown in Fig. 19k. Because of the difference in the backing fibre bundle 


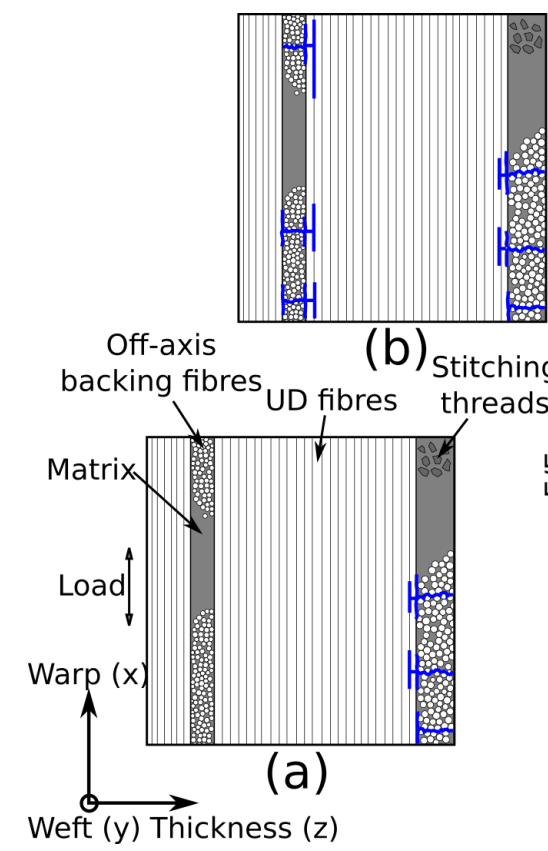

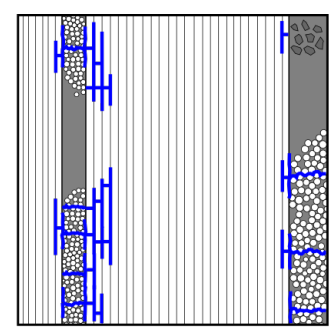

(c)

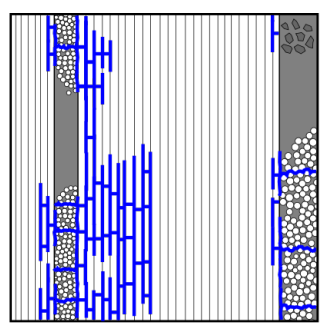

(d)

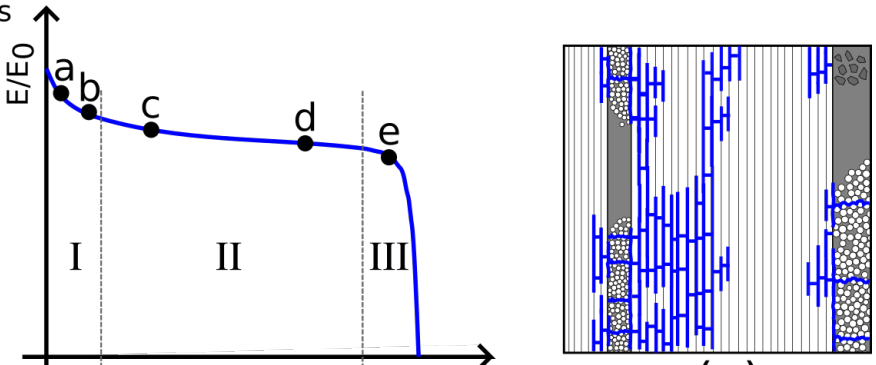

(e)

Figure 19: Slightly modified and expanded version of the damage progression scheme proposed by Zangenberg et al. 17.

thickness the crack density per backing bundle varies. Some fibre fractures might initiate near regions of the stitching thread as well, however as this has not been seen in the X-ray CT images it seems that that the effect of the backing fibre bundles is more significant. Nevertheless, the stitching thread is made from polyester same as the matrix, and therefore they are not visible in the images. During stage II of the fatigue life, the UD fibre fractures gradually grow into the thickness direction of the UD fibre bundles (Fig. 19. to $19 \mathrm{~d}$ ), which is in accordance to the original progression scheme by Zangenberg et al. [17]. Damage regions will start merging (Fig. 19e) and when the remaining UD fibres cannot carry the load anymore final failure occurs.

The scheme presented in Fig. 19 show only 2D views of the damage progression, however as seen from the observations in the current study the UD fibre fractures appear as local 3D phenomena rather than being homogeneously distributed in the UD fibre bundles. This is illustrated in Fig. 20 which shows a sketch of how the UD fibre fractures near a cross-over region of the backing fibre bundles might be distributed at 
a relatively late stage stage of the fatigue life. Several of this type of local damage regions are present in the material where their location and shape depend on the local bundle structure. Furthermore, it is clear that the UD fibre fractures do not severe the UD fibre bundles in 2D fracture planes, and that considering quasi-UD composites in $3 \mathrm{D}$ is important.

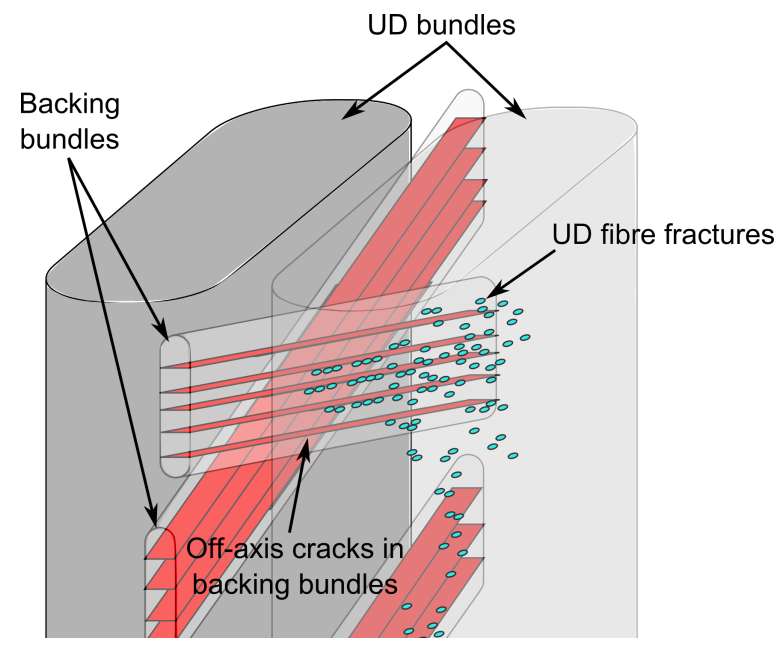

Figure 20: A 3D illustration of how a UD fibre fracture region could look like inside a UD fibre bundle.

To be able to do realistic predictions of the damage initiation and progression in the future, it is necessary to do 3D modelling and also include the effect of the real fibre bundle structure. The presented results and the damage scheme illustrate the importance of establishing methods for modelling the damage progression, that includes the effect of fibre fractures as well. Recent advances within image analysis techniques (e.g. Emerson et al. [39]) have made it possible to extract individual fibres from Xray CT data, and it could be possible to extract the fibre bundle structure by similar means. This allows one to use the actual material structure on different length scales in a finite element model. Therefore, the work carried out through the current study that lead to the damage progression scheme shown in Fig. 19 serves as a good base for establishing a multi-scale X-ray based finite element model. Such an approach makes it possible to both include the real fibre and bundle structure obtained by X-ray CT and the effect of fibre fractures in a future finite element model. The 3D data sets along 
with the monitored damage progression provided by the current study can be used for this purpose.

\section{Conclusion}

In the current study, a method for monitoring and automatically quantifying the off-axis crack density in the backing fibre bundles of a quasi-UD NCF composite tested in fatigue was presented. Fatigue tests were carried out at different strain levels, and the crack density seemed to level out at different values off-axis crack densities. Hence, it was clear that the strain level had an influence on the fatigue damage mechanisms. In addition, the initiation and progression of UD fibre fractures was monitored in two different regions of a quasi-UD composite using an ex-situ X-ray CT fatigue testing approach. By monitoring two different damage regions, it was observed that UD fibre fractures initiated first at the cross-over region of the backing fibre bundles and later at the single backing fibre bundle region. UD fibre fractures were only observed at locations where the backing fibre bundles were in contact with a UD fibre bundle, which indicated that the UD fibre fractures initiate from off-axis cracks in the backing fibre bundles. Although generally not visible in the X-ray CT scans, the off-axis cracks were shown to be present by TWLI. A tension clamp solution was applied to two specimens tested in fatigue and interrupted early and late in the fatigue life to investigate the crack visibility in the X-ray CT scans. These experiments showed that keeping the specimen under load during X-ray CT scanning had a large influence on the crack visibility for the considered material. By applying load, some off-axis cracks also became visible and they were seen to be accompanied by UD fibre fractures highly indicating that these fibre fractures initiated from the off-axis cracks.

\section{Acknowledgements}

Financial support from CINEMA: "the allianCe for ImagiNg of Energy MAterials", DSF-grant no. 1305-00032B under "The Danish Council for Strategic Research" is 
gratefully acknowledged. This research was conducted using mechanical testing equipment from DTU Center for Advanced Structural and Material Testing (CASMAT), Grant No. VKR023193 from Villum Fonden. Additionally we would like to thank LM Wind Power for manufacturing of test specimens. Finally, we would like to thank Steffen Rasmussen and Anthony Fraisse from DTU Wind Energy for their assistance with mechanical tests.

\section{Appendix A. Modified tension clamp}

The tension clamp used in the current study was a modified version of the clamp described in [19] that could fit the smaller specimen geometry used in the current study, as shown in Fig. A.1 The tension clamp was made from two aluminium parts connected by two carbon pins loaded in compression when the clamp was straining the specimen. To attach the tension clamp, the test specimen was first loaded in tension in a regular tensile testing machine. The clamp was then mounted by screws on the strained specimen. When the load was removed from the test machine, the tension clamp kept the gauge section of the specimen loaded. The tension clamp utilises the curvature of the specimen edges to keep the aluminium parts in place and thereby transfer the load with no pressure on the specimen surface. Therefore, if damage is induced to the specimen it will be at the curved part of the specimens outside the gauge section. Visually the specimen did not seem to have suffered significant damage. For additional information on the tension clamp the reader is referred to [19], where a similar clamp was presented for a larger specimen geometry.

\section{Appendix B. Crack counting relative to bundle structure}

In this appendix, some additional details on the crack counting method used in this paper are provided. 


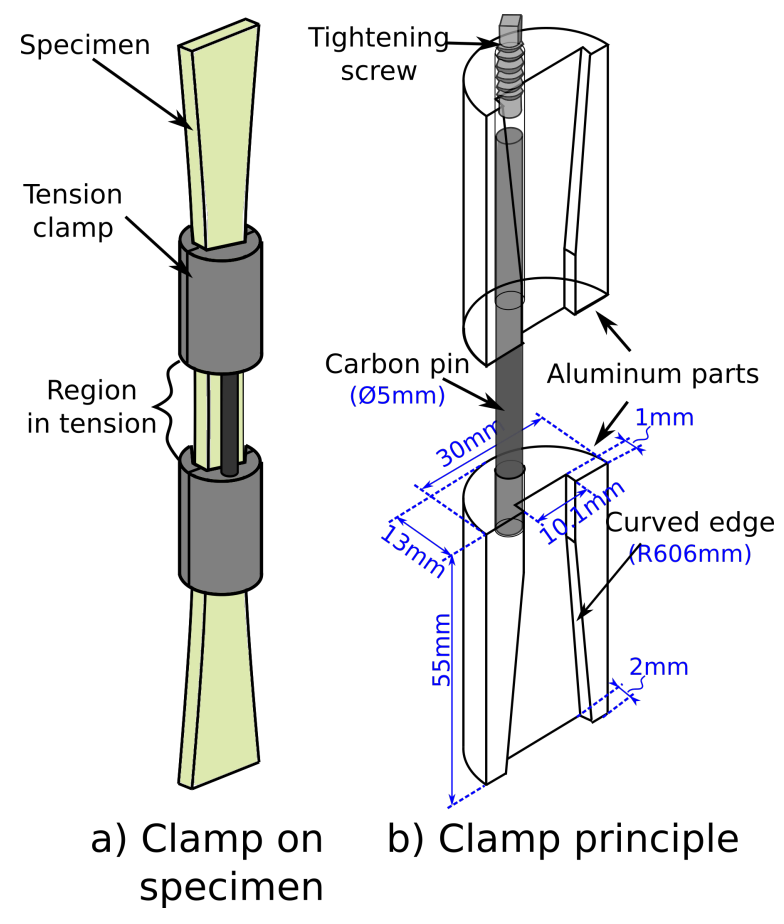

Figure A.1: Illustration of (a) the tension clamp on a specimen and (b) the principle of the clamp with dimensions.

\section{Appendix B.1. Automated crack counting method}

The crack couting process includes three overall steps; image compensation, image filtering, and crack counting. In the image compensation step, all the images are compensated for the deformation when loading the sample and other movements to be able to normalise them relative to the initial image of the unloaded and undamaged specimen. The image filtering step has the purpose of separating the cracks observed in different layers with different fibre orientations. This is done using Gabor-filtering which can be used to highlight the cracks oriented in a specific direction and thereby provide separate images for each crack orientation. The normalised and filtered images for each crack orientation are then thresholded to obtain binary images and the cracks are thinned down to one pixel thick lines. To count the cracks, the images are rotated to be vertically aligned. The cracks are counted by going through the image pixels row by row, and the presence of a black pixel defines the start of a crack. For each crack start, the column belonging to that pixel is searched for additional black pixels connected to 
the crack. Since the cracks are likely not to be perfectly aligned, neighboring columns are also searched if a white pixel is found. The end of a crack is obtained when a white pixel is reached and no black pixels are present in the nearby pixels. The start and end points of the crack are stored, and the counted crack is deleted from the image. This is then repeated until there are no more cracks in the image. Hence, the cracks are counted as straight lines.

Since the algorithm initially was used for laminates with a relatively homogeneous distribution of fibres within each layer, it did not work well with variation in the orientation of the cracks within each layer. The reason is that the algorithm looks for cracks in a specific angle given as input. Since there can be quite some variation in the local orientation angle of the backing fibre bundles in the considered material, it was necessary to modify the algorithm slightly to obtain good results. This was done by automatically determining the average orientation angle of the cracks in each individual backing fibre bundle, and look for cracks in each backing fibre bundle one by one. This modification requires one initial step where the approximate regions of the backing fibre bundles have to be marked manually, however this only has to be done once per sample and only roughly.

\section{Appendix B.2. Extracting the fibre bundle structure}

A simple and less time consuming method than 3D segmentation was established to obtain the projected backing bundle area in 2D as illustrated in Fig. B.2.

Initially, the 3D dataset obtained by X-ray CT was sliced into a stack of 2D images in the $\mathrm{x}-\mathrm{y}$ plane (see Fig. B.2). The 2D images containing the backing fibre bundles were then extracted, made opague (30\%), and combined into an overlay image. In the overlay image, the edges of all the backing bundles then had become visible. The projected backing bundle area was then segmented by manually marking the area in the open source software ImageJ [37]. After applying a threshold to the image, the projected backing bundle area was calculated using the 'Analyze particles' function in ImageJ. 


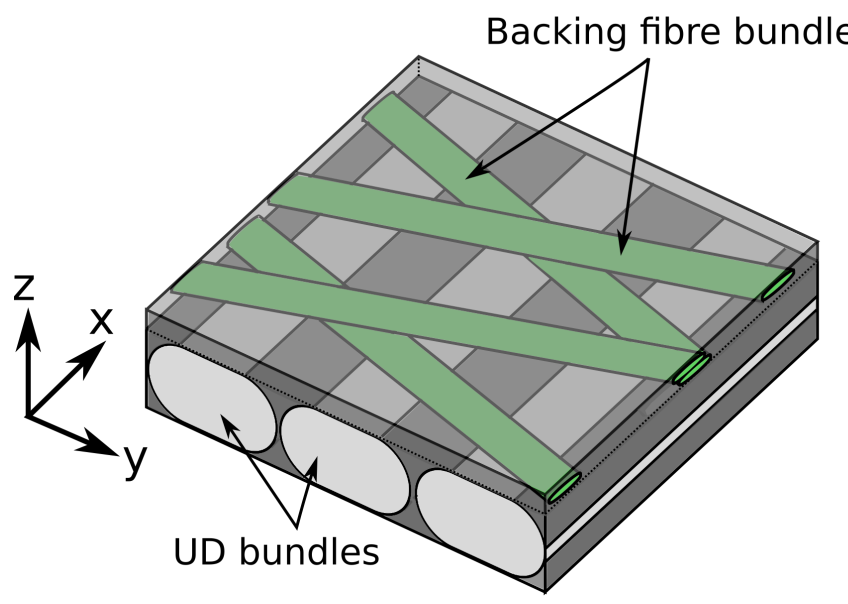

a) Bundle structure $\square$ Projected backing bundle area

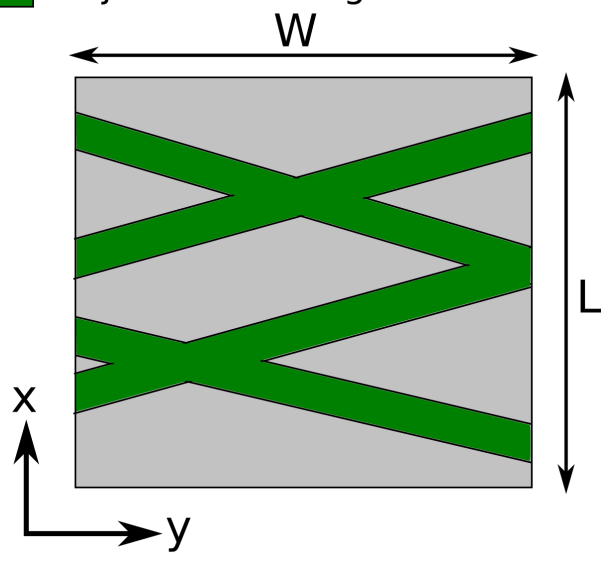

b) Top view of backing bundles (XY-plane)

Figure B.2: Illustration of a) the fibre bundle structure and b) the definition of the projected backing bundle area.

\section{Image overlay of slices containing backing from \\ 3D X-ray CT data}

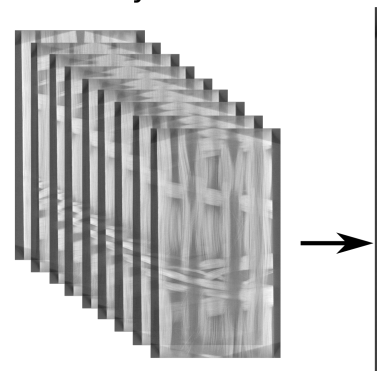

Overlay image

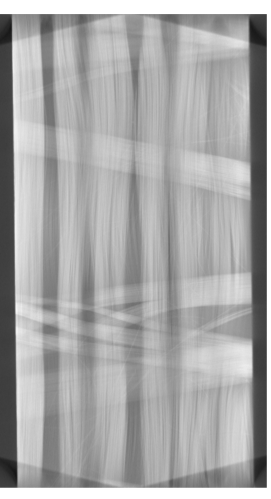

Manual segmentation of projected bundle area Thresholding to get on top of overlay image backing bundle area

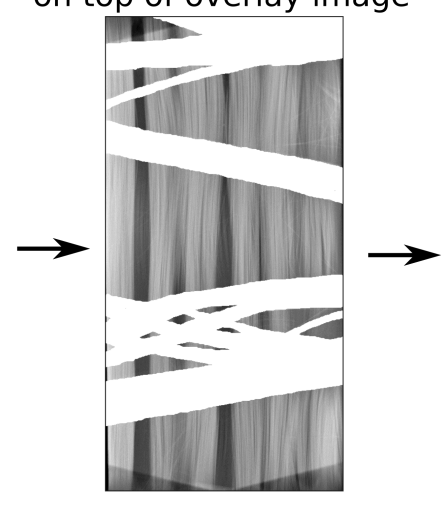

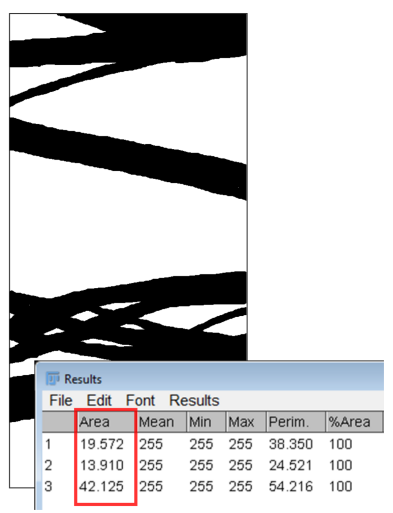

Figure B.3: Sketch of principle for segmentation of projected backing bundle area

The ratio between the backing bundle area and the matrix area is given by Eq. B.1 where $A_{t o t}=L \cdot W$. Fig. B.4 shows the projected backing bundle areas for all the specimens obtained using the above method along with their $\alpha_{L}$ value. It is seen that there is quite some variation.

$$
\alpha_{L}=\frac{A_{b b}}{A_{t o t}-A_{b b}}
$$




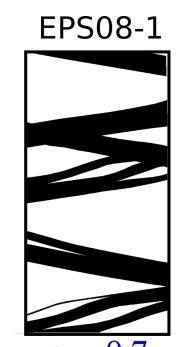

$\alpha_{L}=0.7$

EPS06-4
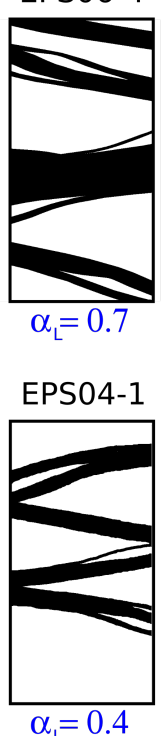
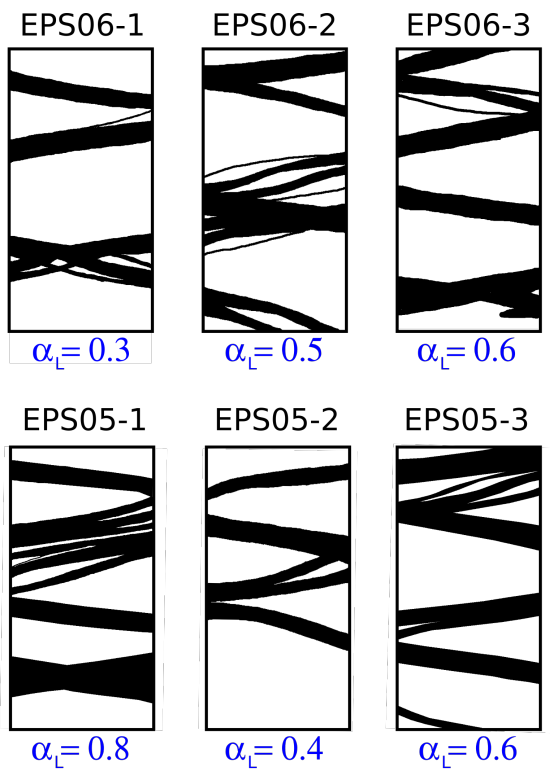

EPS05-3
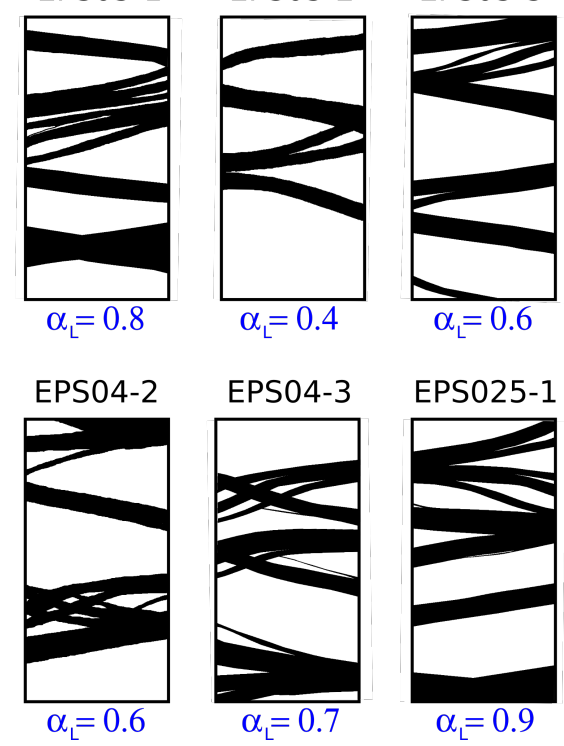

Figure B.4: Projected backing bundle areas for all the tested specimens in the considered area (10x20mm) obtained by X-ray CT. The parts marked in black show the backing bundles. $\alpha_{L}$ is defined by equation B.1.

\section{References}

[1] R. P. L. Nijssen, P. Brøndsted, Fatigue as a design driver for composite wind turbine blades, Advances in Wind Turbine Blade Design and Materials (2013). doi:10.1533/9780857097286.2.175.

[2] K. Vallons, G. Adolphs, P. Lucas, S. V. Lomov, I. Verpoest, Quasi-UD glass fibre NCF composites for wind energy applications: a review of requirements and existing fatigue data for blade materials, Mechanics \& Industry 14 (3) (2013) 175-189. doi:10.1051/meca/2013045. 
[3] P. Brøndsted, H. Lilholt, H. A. Lystrup, Composite materials for wind power turbine blades, Annual Review of Materials Research 35 (2006) 505-538. doi: 10.1146/annurev.matsci.35.100303.110641.

[4] R. P. L. Nijssen, Fatigue life prediction and strength degradation of wind turbine rotor blade composites, PhD Thesis, Delt University of Technology (2006).

[5] J. Tong, F. J. Guild, S. L. Orgin, P. A. Smith, On matrix crack growth in quasiisotropic laminates - I. experimental investigation, Composites Science and Technology 57 (11) (1997) 1527-1535. doi:10.1016/S0266-3538(97)00080-8.

[6] M. Quaresimin, P. A. Carraro, L. P. Mikkelsen, N. Lucato, L. Vivian, P. Brøndsted, B. F. Sørensen, J. Varna, R. Talreja, Damage evolution under cyclic multiaxial stress state: A comparative analysis between glass/epoxy laminates and tubes, Composites: Part B 61 (2014) 282-290. doi:10.1016/j.compositesb.2014.01. 056.

[7] A. Hosoi, Y. Arao, H. Karasawa, H. Kawada, High-cycle fatigue characteristics of quasi-isotropic CFRP laminates, Advanced Composite Materials 16 (2) (2007) 151-166. doi:10.1163/156855107780918964.

[8] J. Zangenberg, The effects of fibre architecture on fatigue life-time of composite materials, PhD Thesis, DTU Wind Energy PhD-0018(EN) (2013).

[9] K. L. Reifsnider, R. Jamison, Fracture of fatigue-loaded composite laminates, International Journal of Fatigue 4 (4) (1982) 187-197. doi:10.1016/0142-1123(82) 90001-9.

[10] R. D. Jamison, K. Schulte, K. L. Reifsnider, W. W. Stinchcomb, Characterization and Analysis of Damage Mechanisms in Tension-Tension Fatigue of Graphite/Epoxy Laminates, Effects of Defects in Composite Materials STP30196S (1984) 21-55. doi:10.1520/STP30196S. 
[11] J. Varna, R. Joffe, N. V. Akshantala, R. Talreja, Damage in composite laminates with off-axis plies, Composites Science and Technology 59 (14) (1999) 2139-2147. doi:10.1016/S0266-3538(99)00070-6.

[12] A. Gagel, D. Lange, K. Schulte, On the relation between crack densities, stiffness degradation, and surface temperature distribution of tensile fatigue loaded glassfibre non-crimp-fabric reinforced epoxy, Composites: Part A 37 (2) (2006) 222-228. doi:10.1016/j.compositesa.2005.03.028.

[13] F. Edgren, D. Mattsson, L. E. Asp, J. Varna, Formation of damage and its effects on non-crimp fabric reinforced composites loaded in tension, Composites Science and Technology 64 (5) (2004) 675-692. doi:10.1016/s0266-3538(03)00292-6.

[14] K. Vallons, G. Adolphs, P. Lucas, S. V. Lomov, I. Verpoest, The influence of the stitching pattern on the internal geometry, quasi-static and fatigue mechanical properties of glass fibre non-crimp fabric composites, Composites: Part A 56 (2014) 272-279. doi:10.1016/j.compositesa.2013.10.015.

[15] S. Adden, P. Horst, Stiffness degradation under fatigue in multiaxially loaded noncrimped-fabrics, International Journal of Fatigue 32 (1) (2010) 108-122. doi: $10.1016 /$ j.ijfatigue.2009.02.002.

[16] P. Horst, S. Adden, Fatigue behavior of non-crimp fabrics, Key Engineering Materials 385-387 (2008) 545-548. doi:10.4028/www.scientific.net/KEM.385-387. 545.

[17] J. Zangenberg, P. Brøndsted, J. W. Gillespie Jr., Fatigue damage propagation in unidirectional glass fibre reinforced composites made of a non-crimp fabric, Journal of Composite Materials 48 (22) (2014) 2711-2727. doi:10.1177/ 0021998313502062 .

[18] K. M. Jespersen, J. Zangenberg, T. Lowe, P. J. Withers, L. P. Mikkelsen, Fatigue damage assessment of uni-directional non-crimp fabric reinforced polyester com- 
posite using x-ray computed tomography, Composites Science and Technology 136 (2016) 94-103. doi:10.1016/j.compscitech.2016.10.006.

[19] K. M. Jespersen, L. P. Mikkelsen, Fatigue damage observed non-destructively in fibre composite coupon test specimens by x-ray ct, IOP Conference Series: Materials Science and Engineering 139 (2016) 012024. doi:10.1088/1757-899X/139/ $1 / 012024$

[20] K. M. Jespersen, Y. Wang, T. Zangenberg, J. Lowe, P. J. Withers, L. P. Mikkelsen, Ex-situ time-lapse X-ray CT study of 3D micro-structural fatigue damage evolution in uni-directional composites, in: 17th European Conference on Composite Materials, Munich, 2016, pp. 1-8.

[21] E. Maire, P. J. Withers, Quantitative X-ray tomography, International Materials Reviews 59 (1) (2014) 1-43. doi:10.1179/1743280413y.0000000023.

[22] P. J. Withers, M. Preuss, Fatigue and damage in structural materials studied by X-Ray tomography, Annual Review of Materials Research 42 (2012) 81-103. doi:10.1146/annurev-matsci-070511-155111.

[23] P. Wright, X. Fu, I. Sinclair, S. M. Spearing, Ultra high resolution computed tomography of damage in notched carbon fiber-epoxy composites, Journal of Composite Materials 42 (19) (2008) 1993-2002. doi:10.1177/0021998308092211.

[24] P. Wright, A. Moffat, I. Sinclair, S. M. Spearing, High resolution tomographic imaging and modelling of notch tip damage in a laminated composite, Composites Science and Technology 70 (10) (2010) 1444-1452. doi:10.1016/j.compscitech. 2010.04 .012 .

[25] A. E. Scott, M. Mavrigirdati, P. Wright, I. Sinclair, S. M. Spearing, In situ fibre fracture measurement in carbon-epoxy laminates using high resolution computed tomography, Composites Science and Technology 71 (12) (2011) 1471-1477. doi: $10.1016 /$ j.compscitech.2011.06.004. 
[26] S. C. Garcea, M. Macrigirdato, A. E. Scott, I. Sinclair, S. M. Spearing, Fatigue micromechanism characterisation in carbon fibre reinforced polymers using synchrotron radiation computed tomography, Composites Science and Technology 99 (2014) 23-30. doi:10.1016/j.compscitech.2014.05.006.

[27] S. C. Garcea, I. Sinclair, S. M. Spearing, In situ synchrotron tomographic evaluation of the effect of toughening strategies on fatigue micromechanisms in carbon fibre reinforced polymers, Composites Science and Technology 109 (2015) 32-39. doi:10.1016/j.compscitech.2015.01.012.

[28] S. C. Garcea, I. Sinclair, S. M. Spearing, Fibre failure assessment in carbon fibre reinforced polymers under fatigue loading by synchrotron X-ray computed tomography, Composites Science and Technology 133 (2016) 157-164. doi: 10.1016/j.compscitech.2016.07.030.

[29] F. Sket, R. Seltzer, J. M. Molina-Aldareguía, C. Gonzalez, J. LLorca, Determination of damage micromechanisms and fracture resistance of glass fiber/epoxy cross-ply laminate by means of X-ray computed microtomography, Composites Science and Technology 72 (2) (2012) 350-359. doi:10.1016/j.compscitech. 2011.11 .025 .

[30] P. J. Schilling, B. P. R. Karedla, A. K. Tatiparthi, M. A. Verges, P. D. Herrington, X-ray computed microtomography of internal damage in fiber reinforced polymer matrix composites, Composites Science and Technology 65 (14) (2005) 2071-2078. doi:10.1016/j.compscitech.2005.05.014.

[31] B. Yu, R. Blanc, C. Soutis, W. P. J., Evolution of damage during the fatigue of 3D woven glass-fibre reinforced composites subjected to tension-tension loading observed by time-lapse X-ray tomography, Composites: Part A 82 (2016) 279-290. doi:10.1016/j.compositesa.2015.09.001.

[32] B. Yu, R. S. Bradley, C. Soutis, P. J. Hogg, P. J. Withers, 2D and 3D imaging 
of fatigue failure mechanisms of 3D woven composites, Composites Science and Technology 77 (2015) 37-49. doi:10.1016/j.compositesa.2015.06.013.

[33] S. Topal, L. Baiocchi, A. D. Crocombe, S. L. Ogin, P. Potluri, P. J. Withers, M. Quaresimin, P. A. Smith, M. C. Poole, A. E. Bogdanovich, Late-stage fatigue damage in a 3D orthogonal non-crimp woven composite: An experimental and numerical study, Composites: Part A 79 (2015) 155-163. doi: $10.1016 / j$.compositesa.2015.08.020.

[34] J. Pazmino, V. Carvelli, S. V. Lomov, Micro-CT analysis of the internal deformed geometry of a non-crimp 3D orthogonal weave E-glass composite reinforcement, Composites Part B: Engineering 65 (2014) 147-157. doi:10.1016/j. compositesb.2013.11.024.

[35] J. A. Glud, J. M. Dulieu-barton, O. T. Thomsen, L. C. T. Overgaard, Automated counting of off-axis tunnelling cracks using digital image processing, Composites Science and Technology 125 (2016) 80-89. doi:10.1016/j.compscitech.2016. 01.019 .

[36] K. M. Jespersen, J. A. Glud, Z. J., A. Hosoi, H. Kawada, L. P. Mikkelsen, Exsitu X-ray computed tomography, tension clamp and in-situ transilluminated white light imaging data of non-crimp fabric based fibre composite under fatigue loading, Data in Brief (Submitted) (2018).

[37] J. Schindelin, I. Arganda-Carreras, E. Frise, V. Kaynig, M. Longair, T. Pietzsch, S. Preibisch, C. Rueden, S. Saalfeld, B. Schmid, J.-Y. Tinevez, D. J. White, V. Hartenstein, K. Eliceiri, P. Tomancak, A. Cardona, Fiji: an open-source platform for biological-image analysis, Nature Methods 9 (7) (2012) 676-682.

[38] J. Tong, Three Stages of Fatigue Crack Growth in GFRP Composite, Journal of Engineering Materials and Technology 123 (2001) 139-143. doi:10.1115/1. 1286234 . 
[39] M. J. Emerson, K. M. Jespersen, A. B. Dahl, K. Conradsen, L. P. Mikkelsen, Individual fibre segmentation from 3D X-ray computed tomography for characterising the fibre orientation in unidirectional composite materials, Composites: Part A (2017). doi:10.1016/j.compositesa.2016.12.028. 